\title{
Surface sediment and phytoplankton diatoms across a trophic gradient in tropical reservoirs: new records for Brazil and São Paulo State
}

\author{
Elaine Cristina Rodrigues Bartozek ${ }^{10,2,3}$, Stéfano Zorzal-Almeida ${ }^{2}$ and Denise C. Bicudo ${ }^{2}$
}

Received: 10.08.2017; accepted: 19.12.2017

\begin{abstract}
Surface sediment and phytoplankton diatoms across a trophic gradient in tropical reservoirs: new records for Brazil and São Paulo State). This,study aimed to inventory the diatom flora of tropical reservoirs from southeastern region of Brazil under oligo- to hypereutrophic conditions. We collected diatom samples from the surface sediment and phytoplankton (summer and winter) in 41 sites (ten reservoirs). Seventy-eight taxa, distributed in 28 genera, were identified in the surface sediment and phytoplankton samples. Nizschia was the most representative genus, with nine species. Six taxa represent new records for Brazil and six for São Paulo State; ten taxa were identified at the genus level and probably represent new species. Additionally, this study contributed with data on the ecology of the species and evidenced the necessity of floristic surveys to improve the knowledge about tropical diatom biodiversity.
\end{abstract}

Keywords: biodiversity, diatom flora, ecology

RESUMO - (Diatomáceas de sedimentos superficiais e fitoplanctônicas ao longo de um gradiente trófico em reservatórios tropicais: novos registros para o Brasil e Estado de São Paulo). Este estudo visou inventariar a flora diatomológica de represas tropicais em condições oligo- a hipereutróficas da região sudeste do Brasil. As amostras abrangeram diatomáceas do sedimento superficial e fitoplâncton (verão e inverno) em 41 locais (dez represas). Setenta e oito táxons, distribuídos em 28 gêneros, foram identificados nas amostras de sedimento superficial e fitoplanctônicas. Nitzschia foi o gênero mais representativo, com um total de nove espécies. Seis táxons representam novas citações para o Brasil e seis para o Estado de São Paulo; dez táxons foram identificados em nível de gênero e provavelmente representam novas espécies. Adicionalmente, este estudo contribuiu com informações sobre a ecologia dessas espécies e permite enfatizar a necessidade de estudos florísticos para ampliar o conhecimento da biodiversidade de diatomáceas tropicais.

Palavras-chave: biodiversidade, ecologia, flora diatomológica

\section{Introduction}

Evidences show that recent species extinctions rates are exceptionally high, suggesting a probably new event of mass extinction due the environmental crisis caused by mankind (Ceballos et al. 2015). Despite the efforts on taxonomic and ecological studies, many species are extinct even before they can be described. The biota of freshwater ecosystems has been highly neglected in studies about biodiversity, especially invertebrates and microorganisms at the tropical region (Dudgeon et al. 2006). Freshwater ecosystems support a high diversity despite their reduced superficial area, therefore being considered hot spots for biodiversity (Strayer \& Dudgeon 2010).
Diatoms are among the most species-rich group of algae and the number of species is around 100,000, including fossil species (Mann \& Vanormelingen 2013). They are an abundant component of primary producers in plankton and benthos, both in marine and freshwaters (Round et al. 1990). Due to their high richness and abundance in aquatic ecosystems, diatoms represent an important account to local and regional biodiversity, which can be accessed by floristic surveys. Furthermore, ecological preferences of many species are relatively well know, making these group widely used to evaluate environmental conditions (e.g., Passy 2007, Bennion et al. 2014, Blanco et al. 2014). However, just about $12 \%$ of the estimated diatom flora is currently described (Julius \& Theriot 2010).

1. Universidade Estadual Paulista "Júlio de Mesquita Filho, Instituto de Biociências, Av. 24A, 1515, 13506-900 Rio Claro, SP, Brazil

2. Instituto de Botânica, Núcleo de Pesquisa em Ecologia, Av. Miguel Estéfano 3687, 04301-902 São Paulo, SP, Brazil

3. Corresponding author: elaine.bartozek@gmail.com 
In recent years, the number of studies about the diatom flora in Brazil has been increasing (e.g., Souza \& Senna 2009, Bertolli et al. 2010, Silva et al. 2010, Bartozek et al. 2013). However the inclusion of surface sediment diatoms is still scarce (Fontana \& Bicudo 2009, 2012, Almeida \& Bicudo 2014, Faustino et al. 2016), and all of them were carried out in the state of São Paulo. Such studies have added several new records to the Brazilian diatom flora and new species to Science (Almeida et al. 2015, Almeida et al. 2016, Marquardt et al. 2016), demonstrating the importance of floristic survey of sediments. Other studies about the diatom flora encompassing lotic and lentic environments have been carried out in the state of São Paulo (Carneiro \& Bicudo 2007, Bere \& Tundisi 2010, Marquardt \& Bicudo 2014, Ferreira \& Bicudo 2017). The present study was carried out in ten reservoirs from three watersheds of São Paulo State (Brazil). To our knowledge, this is a pioneer diatom floristic survey to the study area, except for the unpublished study carried out by Silva (2017). We aimed at improving the knowledge of the diatom flora from surface sediments and phytoplankton, highlighting the new records for Brazil and São Paulo State. Furthermore, this study contributes to the increase of knowledge about biodiversity and ecology of tropical diatoms.

\section{Material and methods}

This study was carried out in the southeastern region of Brazil and comprises three drainage basins in the State of São Paulo (figure 1, table 1). We selected 10 reservoirs located in protected and highly urbanized areas, ranging from oligo- to hypereutrophic conditions and with different uses (recreational, power generation, navigation and public water supply). The reservoirs range from shallow to deep (maximum depth from 2 to $33 \mathrm{~m}$ ) and from small to large (surface area from 0.2 to $241.3 \mathrm{~km}^{2}$ ). We selected three to six sampling sites (table 1) per reservoir depending on size, the main water inputs (main streams) and deepest region of the reservoirs.

We sampled a total of forty-one sites (table 1) during two climatic periods (austral winter and summer) in 2013 and 2014. Water column samples were taken with a van Dorn bottle along the reservoir vertical profile (subsurface, mean depth and $1 \mathrm{~m}$ above the sediments), and mean results of water column were used to characterize each sampling site. Conductivity, $\mathrm{pH}$ and water temperature were measured in field using standard electrodes (Horiba U-50). The analytical methods for dissolved oxygen, dissolved inorganic nitrogen, total nitrogen, orthophosphate, total phosphorus and soluble reactive silica followed Standard Methods (APHA 2005). The reservoirs Trophic State Index (TSI) was calculated according to Lamparelli (2004) based on values of chlorophyll- $a$ (Sartory \& Grobbelaar 1984) and total phosphorus. Integrated water column samples were used to describe the phytoplankton diatom community. Surface sediment diatoms (top $2 \mathrm{~cm}$ ) were sampled only in winter (41 samples) using a UWITEC gravity

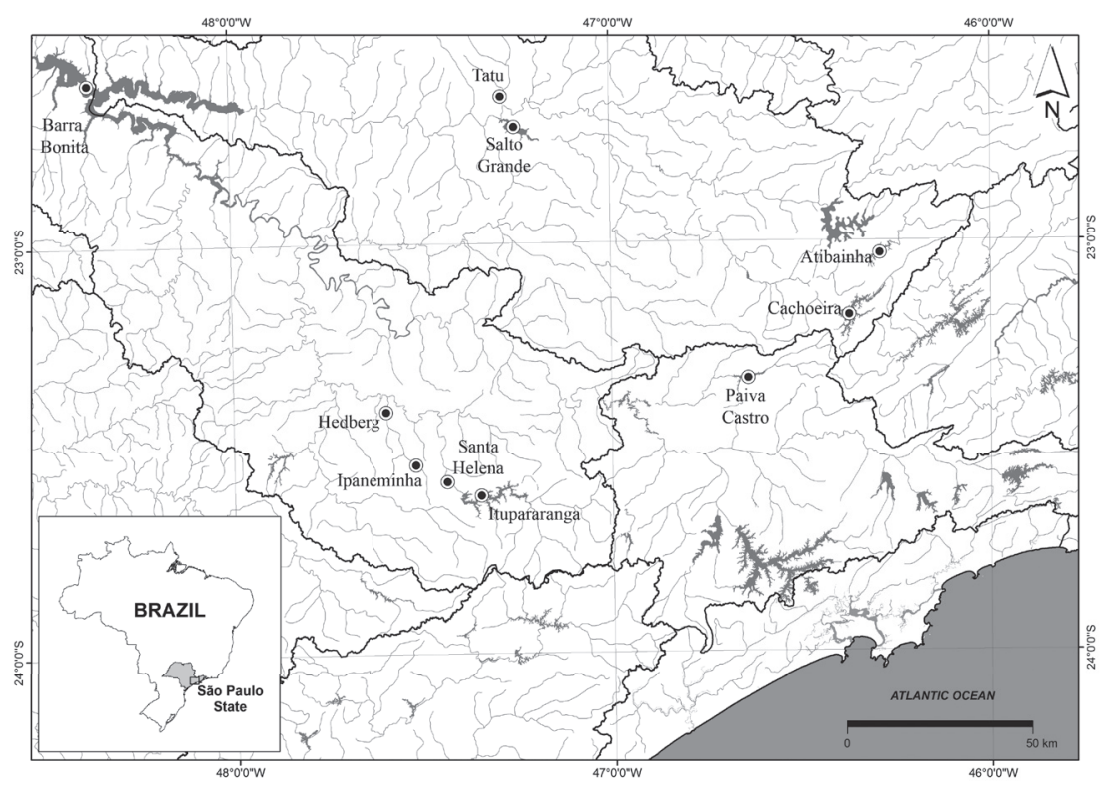

Figure 1. Location of the ten studied reservoirs in southeastern region of Brazil. Black lines represent the boundaries of the basins. Modified from Bicudo et al. (2016). 
Table 1. Watersheds, reservoirs, number and codes of sampling sites and number of samples collected from surface sediments (SS) and phytoplankton (P) in each reservoir.

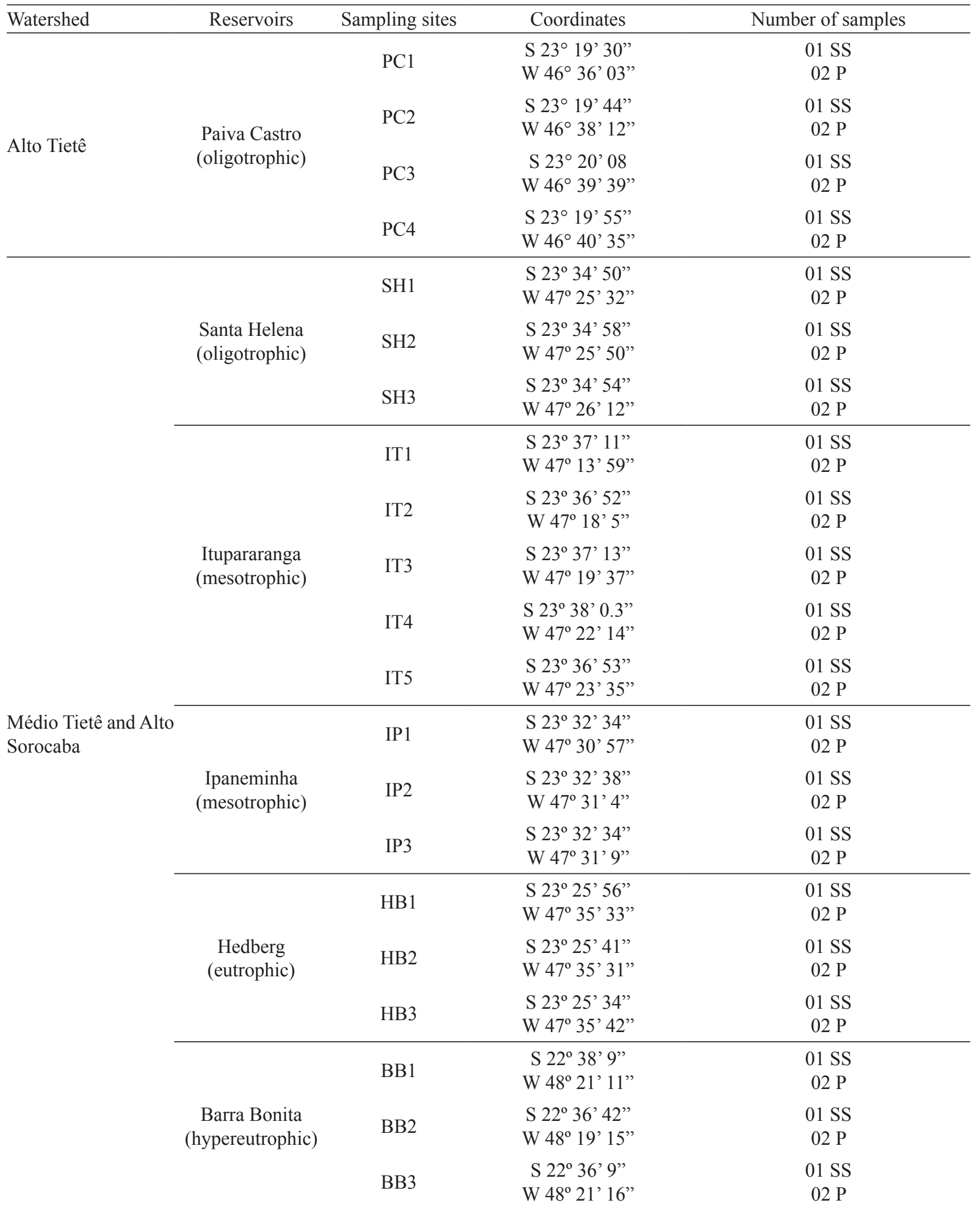


Table 1 (continuation)

\begin{tabular}{|c|c|c|c|c|}
\hline Watershed & Reservoirs & Sampling sites & Coordinates & Number of samples \\
\hline \multirow{2}{*}{$\begin{array}{l}\text { Médio Tietê and Alto } \\
\text { Sorocaba }\end{array}$} & \multirow{2}{*}{$\begin{array}{c}\text { Barra Bonita } \\
\text { (hypereutrophic) }\end{array}$} & BB4 & $\begin{array}{c}\mathrm{S} 22^{\circ} 34^{\prime} 9^{\prime \prime} \\
\mathrm{W} 48^{\circ} 24^{\prime} 31^{\prime \prime}\end{array}$ & $\begin{array}{c}01 \mathrm{SS} \\
02 \mathrm{P}\end{array}$ \\
\hline & & BB5 & $\begin{array}{l}\text { S } 22^{\circ} 31^{\prime} 56^{\prime \prime} \\
\text { W } 48^{\circ} 27^{\prime} 37^{\prime \prime}\end{array}$ & $\begin{array}{l}01 \mathrm{SS} \\
02 \mathrm{P}\end{array}$ \\
\hline \multirow{18}{*}{$\begin{array}{l}\text { Piracicaba, Capivari } \\
\text { and Jundiaí }\end{array}$} & \multirow{6}{*}{$\begin{array}{l}\text { Atibainha } \\
\text { (oligotrophic) }\end{array}$} & AT1 & $\begin{array}{l}\text { S } 23^{\circ} 08^{\prime} 50^{\prime \prime} \\
\text { W } 46^{\circ} 18^{\prime} 50^{\prime \prime}\end{array}$ & $\begin{array}{l}01 \mathrm{SS} \\
02 \mathrm{P}\end{array}$ \\
\hline & & AT2 & $\begin{array}{l}\text { S } 23^{\circ} 09^{\prime} 42^{\prime \prime} \\
\text { W } 46^{\circ} 21^{\prime} 44^{\prime \prime}\end{array}$ & $\begin{array}{l}01 \mathrm{SS} \\
02 \mathrm{P}\end{array}$ \\
\hline & & AT3 & $\begin{array}{l}\mathrm{S} 23^{\circ} 11^{\prime} 11^{\prime \prime} \\
\text { W } 46^{\circ} 22^{\prime} 51^{\prime \prime}\end{array}$ & $\begin{array}{l}01 \mathrm{SS} \\
02 \mathrm{P}\end{array}$ \\
\hline & & AT4 & $\begin{array}{l}\mathrm{S} 23^{\circ} 10^{\prime} 31^{\prime \prime} \\
\mathrm{W} 46^{\circ} 23^{\prime} 29^{\prime \prime}\end{array}$ & $\begin{array}{l}01 \mathrm{SS} \\
02 \mathrm{P}\end{array}$ \\
\hline & & AT5 & $\begin{array}{l}\text { S } 23^{\circ} 12^{\prime} 46^{\prime \prime} \\
\text { W } 46^{\circ} 22^{\prime} 54^{\prime \prime}\end{array}$ & $\begin{array}{c}01 \mathrm{SS} \\
02 \mathrm{P}\end{array}$ \\
\hline & & AT6 & $\begin{array}{l}\mathrm{S} 23^{\circ} 10^{\prime} 46^{\prime \prime} \\
\text { W } 46^{\circ} 21^{\prime} 25^{\prime \prime}\end{array}$ & $\begin{array}{l}01 \mathrm{SS} \\
02 \mathrm{P}\end{array}$ \\
\hline & \multirow{5}{*}{$\begin{array}{c}\text { Cachoeira } \\
\text { (oligotrophic) }\end{array}$} & CA1 & $\begin{array}{l}\text { S } 23^{\circ} 00^{\prime} 06^{\prime \prime} \\
\text { W } 46^{\circ} 16^{\prime} 05^{\prime \prime}\end{array}$ & $\begin{array}{l}01 \mathrm{SS} \\
02 \mathrm{P}\end{array}$ \\
\hline & & $\mathrm{CA} 2$ & $\begin{array}{l}\text { S } 23^{\circ} 00^{\prime} 37^{\prime \prime} \\
\text { W } 46^{\circ} 17^{\prime} 11^{\prime \prime}\end{array}$ & $\begin{array}{c}01 \mathrm{SS} \\
02 \mathrm{P}\end{array}$ \\
\hline & & CA3 & $\begin{array}{l}\text { S } 23^{\circ} 01^{\prime} 56^{\prime \prime} \\
\text { W } 46^{\circ} 17^{\prime} 20^{\prime \prime}\end{array}$ & $\begin{array}{c}01 \mathrm{SS} \\
02 \mathrm{P}\end{array}$ \\
\hline & & CA4 & $\begin{array}{l}\text { S } 23^{\circ} 03^{\prime} 00^{\prime \prime} \\
\text { W } 46^{\circ} 19^{\prime} 07^{\prime \prime}\end{array}$ & $\begin{array}{c}01 \mathrm{SS} \\
02 \mathrm{P}\end{array}$ \\
\hline & & CA5 & $\begin{array}{l}\mathrm{S} 23^{\circ} 04^{\prime} 11^{\prime \prime} \\
\mathrm{W} 46^{\circ} 18^{\prime} 41^{\prime \prime}\end{array}$ & $\begin{array}{l}01 \mathrm{SS} \\
02 \mathrm{P}\end{array}$ \\
\hline & \multirow{3}{*}{$\begin{array}{c}\text { Tatu } \\
\text { (mesotrophic) }\end{array}$} & TU1 & $\begin{array}{l}\mathrm{S} 22^{\circ} 38^{\prime} 45^{\prime \prime} \\
\text { W } 47^{\circ} 17^{\prime} 09^{\prime \prime}\end{array}$ & $\begin{array}{l}01 \mathrm{SS} \\
02 \mathrm{P}\end{array}$ \\
\hline & & TU2 & $\begin{array}{l}\text { S } 22^{\circ} 39^{\prime} 17^{\prime \prime} \\
\text { W } 47^{\circ} 17^{\prime} 01^{\prime \prime}\end{array}$ & $\begin{array}{l}01 \mathrm{SS} \\
02 \mathrm{P}\end{array}$ \\
\hline & & TU3 & $\begin{array}{l}\text { S } 22^{\circ} 39^{\prime} 36^{\prime \prime} \\
\text { W } 47^{\circ} 16^{\prime} 45^{\prime \prime}\end{array}$ & $\begin{array}{l}01 \mathrm{SS} \\
02 \mathrm{P}\end{array}$ \\
\hline & \multirow{4}{*}{$\begin{array}{l}\text { Salto Grande } \\
\text { (eutrophic) }\end{array}$} & SG1 & $\begin{array}{l}\text { S } 22^{\circ} 43^{\prime} 43^{\prime \prime} \\
\text { W } 47^{\circ} 13^{\prime} 56^{\prime \prime}\end{array}$ & $\begin{array}{l}01 \mathrm{SS} \\
02 \mathrm{P}\end{array}$ \\
\hline & & SG2 & $\begin{array}{l}\text { S } 22^{\circ} 42^{\prime} 59^{\prime \prime} \\
\text { W } 47^{\circ} 14^{\prime} 26^{\prime \prime}\end{array}$ & $\begin{array}{c}01 \mathrm{SS} \\
02 \mathrm{P}\end{array}$ \\
\hline & & SG3 & $\begin{array}{l}\text { S } 22^{\circ} 43^{\prime} 05^{\prime \prime} \\
\text { W } 47^{\circ} 16^{\prime} 02^{\prime \prime}\end{array}$ & $\begin{array}{l}01 \mathrm{SS} \\
02 \mathrm{P}\end{array}$ \\
\hline & & SG4 & $\begin{array}{l}\text { S } 22^{\circ} 42^{\prime} 04^{\prime \prime} \\
\text { W } 47^{\circ} 16^{\prime} 51^{\prime \prime}\end{array}$ & $\begin{array}{l}01 \mathrm{SS} \\
02 \mathrm{P}\end{array}$ \\
\hline
\end{tabular}


corer. Each sampling site was sampled in triplicate to compound the site spatial heterogeneity.

For diatom analyses, samples were digested using hydrogen peroxide $\left(\mathrm{H}_{2} \mathrm{O}_{2} 35 \%\right)$ and hydrochloric acid ( $\mathrm{HCl} 37 \%$ ) according to Battarbee et al. (2001). Permanent slides were prepared using Naphrax ${ }^{\circledR}$ as inclusion medium. Optical observations, measurements and micrographs were taken at a magnification of $1000 \times$ with a Zeiss Axioskop 2 plus microscope equipped with phase contrast and Axiocam ERc5s high-resolution digital camera. Taxonomy and nomenclature followed specific publications (e.g., Lange-Bertalot 1993, Metzeltin et al. 1998, LangeBertalot et al. 2011) and the on-line catalogue of valid names (site of California Academy of Sciences 2012). The classification systems followed Medlin \& Kaczmarska (2004) for supra-ordinal taxa and Round et al. (1990) for subordinal taxa, except for genera published after to this work.

Diatoms were quantified at a magnification of $1000 \times$ and until reaching a minimum of 400 valves per slide (Battarbee et al. 2001), and at least $90 \%$ in counting efficiency (Pappas \& Stoermer 1996). We included those taxa with relative abundance $\geq 2 \%$ in at least one sampling site and indicated the new records for Brazil and São Paulo state after consulting the published literature (books and articles). Descriptions, relevant taxonomical and ecological comments were provided for taxa identified in genus level. Morphometric information is provided for the new records for Brazil and São Paulo State and for genus level taxa (L: length; W: width; S: striae; A: areolae; F: fibulae). Samples were deposited at the "Herbário Científico do Estado Maria Eneyda P. Kauffmann Fidalgo" (SP), Brazil (SP469229 to SP467313, SP469453, SP469454 and SP456488 to SP469523).

\section{Results and Discussion}

The main environmental features of the study area are available in table 2 . A total of seventy-eight taxa presented relative abundance $\geq 2 \%$, including two non-typical varieties, distributed in 28 genera (table 3). Surface sediment and phytoplankton contributed, respectively, with 60 and 70 taxa. Nitzschia Hassal was the genera with higher number of taxa (nine species) followed by Achnanthidium Kützing (eight species), Aulacoseira Thwaites (eight species) and Fragilaria Lyngbye (eight species). Aulacoseira ambigua and A. granulata var. angustissima were the most spread species in the surface sediment, occurring in 100\% of samples and with the highest abundance in eutrophic conditions (63.6\% and $63.7 \%$, respectively). The most frequent species in the phytoplankton were Aulacoseira ambigua and Discostella stelligera, both with an occurrence of $90.2 \%$ in samples and higher abundances in oligo- and mesotrophic and meso- eutrotrophic conditions, respectively $(63.6 \%$ and 92.0\%). All inventoried taxa and their relative abundances according to the trophic state are presented in table 3. Taxa relative abundance according to surface sediment and phytoplankton assemblages are available in table 4 . Six taxa are new records for Brazil and six for São Paulo State. They are marked with one and two asterisks, respectively. Ten taxa were identified in genus level because they were not found in literature, and probably represent undescribed species. Those taxa are presented below.

\section{Fragilariaceae Greville}

\section{Fragilaria Lyngbye}

*Fragilaria grunowii Lange-Bertalot \& Ulrich, Lauterbornia 78, p. 22-27, pl. 9, fig. 1-12, pl. 10, fig. 1-10, 2014.

Figures 2-4

$\mathrm{L}: 68.5-178.0 \mu \mathrm{m} ; \mathrm{W}: 2.5-3.4 \mu \mathrm{m} ; \mathrm{S}: 13-17$ in $10 \mu \mathrm{m}$.

Short and narrow specimens of $F$. grunowii may be mistaken with large specimens of Fragilaria tenuissima Lange-Bertalot \& Ulrich. However, the specimens found in this study are wider than the type population of $F$. tenuissima (W: 1.6-2.8 $\mu \mathrm{m}$; LangeBertalot \& Ulrich 2014). Specimens shorter than F. grunowii type population (L: $100.0-380.0 \mu \mathrm{m}$ ) were presently found, increasing the length range for this species. Silva (2017) recorded this species in surface sediment and planktonic samples in six of the reservoirs included in this study. This author estimated that this species has preference for slightly acid $(\mathrm{pH}$ : 6.8) and low nutrient content waters (total nitrogen: $417.7 \mu \mathrm{g} \mathrm{L}^{-1}$ and total phosphorus: $\left.15.0 \mu \mathrm{g} \mathrm{L}^{-1}\right)$. In this study, considering surface sediment samples, F. grunowii was found in one mesotrophic reservoir (Itupararanga, maximum abundance: $2.7 \%$ ), and, considering planktonic samples, it was found in three reservoirs ranging from oligo- to eutrophic conditions (Santa Helena, Itupararanga and Hedberg, maximum abundance: $2.9 \%$ ), but with higher frequency of occurrence in mesotrophic waters (37.1\% of samples). This is the second register of the species in Brazil and the first published taxonomical register of this species. 
Table 2. Mean and standard deviation of environmental variables measured in the ten sampled reservoirs $(n=41)$ in summer and winter. Secchi: water transparency, Temp: water temperature, Cond: conductivity, DO: dissolved oxygen, DIN: dissolved inorganic nitrogen, TN: total nitrogen, $\mathrm{PO}_{4}$ : orthophosphate, TP: total phosphorous, SRS: soluble reactive silica, Chl- $a$ : chlorophyll- $a$.

\begin{tabular}{|c|c|c|c|c|c|c|}
\hline \multirow{2}{*}{ Variables } & \multicolumn{2}{|c|}{ Summer } & \multirow{2}{*}{$\begin{array}{l}\text { Standard } \\
\text { deviation }\end{array}$} & \multicolumn{2}{|c|}{ Winter } & \multirow{2}{*}{ Standard deviation } \\
\hline & Range & Mean & & Range & Mean & \\
\hline Depth (m) & $2.0-24.0$ & 10.7 & 5.8 & $1.5-25.2$ & 11.0 & 6.3 \\
\hline Secchi (m) & $0.3-3.2$ & 1.2 & 0.8 & $0.4-2.9$ & 1.5 & 0.6 \\
\hline Temp $\left({ }^{\circ} \mathrm{C}\right)$ & $21.0-29.7$ & 25.7 & 1.9 & $11.4-26.1$ & 17.8 & 3.7 \\
\hline $\mathrm{pH}$ & $5.8-9.6$ & 7.2 & 1.0 & $6.2-9.5$ & 7.2 & 0.9 \\
\hline Cond $\left(\mu \mathrm{S} \mathrm{cm}^{-1}\right)$ & $18.0-361.0$ & 114.1 & 98.5 & $38.0-481.0$ & 151.3 & 139.9 \\
\hline $\mathrm{DO}\left(\mathrm{mg} \mathrm{L}^{-1}\right)$ & $3.7-12.7$ & 6.3 & 1.7 & $2.4-12.2$ & 7.6 & 1.8 \\
\hline $\operatorname{DIN}\left(\mu \mathrm{g} \mathrm{L}^{-1}\right)$ & $23.0-4968.0$ & 536.6 & 1066.0 & $69.5-7548.2$ & 811.8 & 1362.1 \\
\hline $\mathrm{TN}\left(\mu \mathrm{g} \mathrm{L}^{-1}\right)$ & $67.0-9859.1$ & 1337.9 & 2262.5 & $145.9-8136.6$ & 1307.5 & 1500.1 \\
\hline $\mathrm{PO}_{4}\left(\mu \mathrm{g} \mathrm{L}^{-1}\right)$ & $4.0-90.0$ & 12.8 & 17.6 & $4.0-428.1$ & 32.3 & 74.7 \\
\hline $\mathrm{TP}\left(\mu \mathrm{g} \mathrm{L}^{-1}\right)$ & $6.9-567.7$ & 68.4 & 124.8 & $6.5-590.6$ & 77.3 & 124.9 \\
\hline $\mathrm{SRS}\left(\mathrm{mg} \mathrm{L}^{-1}\right)$ & $2.6-6.6$ & 3.8 & 0.9 & $1.0-5.7$ & 3.5 & 1.6 \\
\hline Chl- $a\left(\mu \mathrm{g} \mathrm{L}^{-1}\right)$ & $0.9-363.9$ & 32.2 & 66.5 & $0.5-518.5$ & 38.9 & 92.9 \\
\hline Trophic State Index (TSI) & $48.2-72.8$ & 56.0 & 7.1 & $45.7-75.3$ & 55.8 & 8.2 \\
\hline
\end{tabular}

Table 3. Frequency of occurrence ( $f$ ), and minimum (min) and maximum (max) abundance of diatoms in the sampling sites, according to the trophic state. - : taxon did not occur in sample; taxa in bold are detailed in the present study; *: first record for Brazil; **: first record for São Paulo State.

\begin{tabular}{|c|c|c|c|c|c|c|c|c|c|}
\hline \multirow{2}{*}{ Species } & \multicolumn{3}{|c|}{ Oligotrophic } & \multicolumn{3}{|c|}{ Mesotrophic } & \multicolumn{3}{|c|}{ Eutrophic } \\
\hline & $f$ & $\min$ & $\max$ & $f$ & $\min$ & $\max$ & $f$ & $\min$ & $\max$ \\
\hline $\begin{array}{l}\text { Achnanthidium catenantum (Bily \& Marvan) } \\
\text { Lange-Bertalot }\end{array}$ & 68.0 & 0.00 & 18.38 & 60.0 & 0.00 & 15.31 & 42.1 & 0.0 & 13.6 \\
\hline Achnanthidium exiguum (Grunow) Czarnecki & 50.0 & 0.00 & 26.80 & 20.0 & 0.00 & 25.58 & 5.3 & 0.0 & 0.2 \\
\hline *Achnanthidium jackii Rabenhorst & 76.0 & 0.00 & 6.57 & 37.1 & 0.00 & 6.84 & 34.2 & 0.0 & 24.7 \\
\hline *Achnanthidium lineare Smith & - & - & - & 14.3 & 0.00 & 6.47 & 5.3 & 0.0 & 0.8 \\
\hline $\begin{array}{l}\text { **Achnanthidium cf. macrocephalum } \\
\text { (Hustedt) Round \& Bukhtiyarova }\end{array}$ & 30.0 & 0.00 & 2.58 & 20.0 & 0.00 & 3.00 & 13.2 & 0.0 & 17.1 \\
\hline $\begin{array}{l}\text { Achnanthidium minutissimum (Kützing) } \\
\text { Czarnecki }\end{array}$ & 72.0 & 0.00 & 17.33 & 71.4 & 0.00 & 22.93 & 31.6 & 0.0 & 3.4 \\
\hline $\begin{array}{l}\text { Achnanthidium tropicocatenatum Marquardt } \\
\text { et al. }\end{array}$ & - & - & - & 11.4 & 0.00 & 44.04 & 10.5 & 0.0 & 2.9 \\
\hline Achnanthidium sp. & 2.0 & 0.00 & 0.97 & 17.1 & 0.00 & 6.28 & 5.3 & 0.0 & 0.9 \\
\hline Asterionella formosa Hassal & - & - & - & 2.9 & 0.00 & 0.21 & 13.2 & 0.0 & 2.3 \\
\hline Aulacoseira ambigua (Grunow) Simonsen & 96.0 & 0.00 & 20.90 & 97.1 & 0.00 & 44.05 & 86.8 & 0.0 & 63.6 \\
\hline Aulacoseira calypsi Tremarin et al. & 2.0 & 0.00 & 0.44 & 14.3 & 0.00 & 4.05 & 5.3 & 0.0 & 1.1 \\
\hline $\begin{array}{l}\text { Aulacoseira granulata var. angustissima } \\
\text { (Müller) Simonsen }\end{array}$ & 84.0 & 0.00 & 8.27 & 91.4 & 0.00 & 47.23 & 97.4 & 0.0 & 64.0 \\
\hline $\begin{array}{l}\text { Aulacoseira granulata var. granulata } \\
\text { (Ehrenberg) Simonsen }\end{array}$ & 82.0 & 0.00 & 15.42 & 85.7 & 0.00 & 22.43 & 94.7 & 0.0 & 88.4 \\
\hline
\end{tabular}


Table 3 (continuation)

\begin{tabular}{|c|c|c|c|c|c|c|c|c|c|}
\hline \multirow{2}{*}{ Species } & \multicolumn{3}{|c|}{ Oligotrophic } & \multicolumn{3}{|c|}{ Mesotrophic } & \multicolumn{3}{|c|}{ Eutrophic } \\
\hline & $f$ & $\min$ & $\max$ & $f$ & $\min$ & $\max$ & $f$ & $\min$ & $\max$ \\
\hline $\begin{array}{l}\text { Aulacoseira herzogii (Lemmermann) } \\
\text { Simonsen }\end{array}$ & 4.0 & 0.00 & 0.94 & 14.3 & 0.00 & 1.22 & 18.4 & 0.0 & 2.5 \\
\hline Aulacoseira tenella (Nygaard) Simonsen & 90.0 & 0.00 & 83.95 & 28.6 & 0.00 & 34.24 & 18.4 & 0.0 & 18.9 \\
\hline Aulacoseria veraluciae Tremarin et al. & - & - & - & - & - & - & 2.6 & 0.0 & 2.1 \\
\hline Brachysira brebissonii Ross & - & - & - & - & - & - & 2.6 & 0.0 & 3.1 \\
\hline Brachysira microcephala (Grunow) Compère & 52.0 & 0.00 & 3.84 & 37.1 & 0.00 & 3.46 & 23.7 & 0.0 & 2.0 \\
\hline Brashysira sp. & 16.0 & 0.00 & 5.28 & 5.7 & 0.00 & 0.75 & - & - & - \\
\hline Cyclotella meneghiniana Kützing & 26.0 & 0.00 & 1.23 & 57.1 & 0.00 & 3.62 & 89.5 & 0.0 & 68.5 \\
\hline${ }^{* *}$ Cymbella affinis var. neoprocera Silva & 2.0 & 0.00 & 0.40 & 22.9 & 0.00 & 6.91 & 10.5 & 0.0 & 0.9 \\
\hline $\begin{array}{l}\text { Cymbopleura naviculiformis (Auerswald ex } \\
\text { Heiberg) Krammer }\end{array}$ & 2.0 & 0.00 & 0.40 & 22.9 & 0.00 & 3.08 & 5.3 & 0.0 & 1.1 \\
\hline Diadesmis confervacea Kützing & 4.0 & 0.00 & 0.67 & 14.3 & 0.00 & 7.45 & 34.2 & 0.0 & 8.5 \\
\hline $\begin{array}{l}\text { Discostella pseudostelligera (Hustedt) Houk } \\
\text { \& Klee }\end{array}$ & 10.0 & 0.00 & 48.95 & 17.1 & 0.00 & 46.80 & 15.8 & 0.0 & 9.5 \\
\hline $\begin{array}{l}\text { Discostella stelligera (Cleve \& Grunow) Houk } \\
\text { \& Klee }\end{array}$ & 100.0 & 0.60 & 65.92 & 97.1 & 0.00 & 91.99 & 73.7 & 0.0 & 33.4 \\
\hline Encyonema silesiacum (Bleisch) Mann & 60.0 & 0.00 & 7.11 & 28.6 & 0.00 & 5.49 & 18.4 & 0.0 & 3.0 \\
\hline Encyonema sp. & 26.0 & 0.00 & 2.19 & 5.7 & 0.00 & 2.49 & - & - & - \\
\hline $\begin{array}{l}\text { *Encyonopsis thienemannii (Hustedt) } \\
\text { Krammer }\end{array}$ & 4.0 & 0.00 & 3.63 & 5.7 & 0.00 & 0.98 & - & - & - \\
\hline Eunotia botulitropica Wetzel \& Costa & 48.0 & 0.00 & 5.74 & 28.6 & 0.00 & 17.65 & 15.8 & 0.0 & 2.6 \\
\hline $\begin{array}{l}\text { Eunotia desmogonioides Metzeltin \& Lange- } \\
\text { Bertalot }\end{array}$ & - & - & - & 2.9 & 0.00 & 4.41 & - & - & - \\
\hline Eunotia intricans Lange-Bertalot \& Metzeltin & 32.0 & 0.00 & 4.38 & 37.1 & 0.00 & 28.64 & 5.3 & 0.0 & 2.3 \\
\hline Eunotia longicamelus Costa et al. & 14.0 & 0.00 & 18.78 & 17.1 & 0.00 & 3.67 & - & - & - \\
\hline Eunotia meridiana Metzeltin \& Lange-Bertalot & 12.0 & 0.00 & 2.29 & 5.7 & 0.00 & 2.00 & - & - & - \\
\hline Fragilaria aquaplus Lange-Bertalot \& Ulrich & 4.0 & 0.00 & 1.62 & 14.3 & 0.00 & 2.29 & 15.8 & 0.0 & 18.2 \\
\hline Fragilaria crotonensis Kitton & 24.0 & 0.00 & 2.75 & 11.4 & 0.00 & 2.73 & 2.6 & 0.0 & 0.4 \\
\hline *Fragilaria grunowii Lange-Bertalot \& Ulrich & 4.0 & 0.00 & 1.20 & 37.1 & 0.00 & 2.73 & 21.1 & 0.0 & 4.5 \\
\hline $\begin{array}{l}\text { Fragilaria longifusiformis (Hains \& Sebring) } \\
\text { Siver et al. }\end{array}$ & 16.0 & 0.00 & 9.00 & 31.4 & 0.00 & 12.27 & 13.2 & 0.0 & 14.1 \\
\hline Fragilaria parva (Grunow) Tuji \& Williams & 10.0 & 0.00 & 0.98 & 5.7 & 0.00 & 8.96 & - & - & - \\
\hline Fragilaria spectra Almeida et al. & 4.0 & 0.00 & 19.45 & 22.9 & 0.00 & 38.18 & 10.5 & 0.0 & 46.4 \\
\hline $\begin{array}{l}\text { **Fragilaria tenera var. nanana (Lange- } \\
\text { Bertalot) Lange-Bertalot \& Ulrich }\end{array}$ & - & - & - & 25.7 & 0.00 & 30.42 & 7.9 & 0.0 & 26.5 \\
\hline Fragilaria sp. & 22.0 & 0.00 & 7.52 & 20.0 & 0.00 & 18.36 & - & - & - \\
\hline Geissleria punctifera (Hustedt) Metzeltin et al. & 12.0 & 0.00 & 2.99 & 2.9 & 0.00 & 0.21 & - & - & - \\
\hline $\begin{array}{l}\text { Geissleria lateropunctata (Wallace) Potapova } \\
\& \text { Winter }\end{array}$ & 42.0 & 0.00 & 7.65 & 20.0 & 0.00 & 1.49 & - & - & - \\
\hline Gomphonema hawaiense Reichardt & 16.0 & 0.00 & 0.74 & 11.4 & 0.00 & 1.33 & 5.3 & 0.0 & 2.0 \\
\hline
\end{tabular}


Table 3 (continuation)

\begin{tabular}{|c|c|c|c|c|c|c|c|c|c|}
\hline \multirow{2}{*}{ Species } & \multicolumn{3}{|c|}{ Oligotrophic } & \multicolumn{3}{|c|}{ Mesotrophic } & \multicolumn{3}{|c|}{ Eutrophic } \\
\hline & $f$ & $\min$ & $\max$ & $f$ & $\min$ & $\max$ & $f$ & $\min$ & $\max$ \\
\hline Gomphonema lagenula Kützing & 40.0 & 0.00 & 1.50 & 34.3 & 0.00 & 11.03 & 21.1 & 0.0 & 3.2 \\
\hline${ }^{* * G o m p h o n e m a ~ n a v i c u l o i d e s}$ Smith & 10.0 & 0.00 & 2.94 & 14.3 & 0.00 & 2.49 & - & - & - \\
\hline Gomphonema parvulum (Kützing) Kützing & 10.0 & 0.00 & 2.06 & 5.7 & 0.00 & 3.23 & 5.3 & 0.0 & 0.3 \\
\hline Hantzschia amphioxys (Ehrenberg) Grunow & 4.0 & 0.00 & 1.96 & 5.7 & 0.00 & 3.46 & - & - & - \\
\hline Humidophila contenta (Grunow) Lowe & 58.0 & 0.00 & 8.37 & 40.0 & 0.00 & 3.63 & 18.4 & 0.0 & 2.8 \\
\hline Navicula cryptocephala Kützing & 6.0 & 0.00 & 1.72 & 34.3 & 0.00 & 14.38 & 10.5 & 0.0 & 18.1 \\
\hline Navicula cryptotenella Lange-Bertalot & 4.0 & 0.00 & 1.36 & 20.0 & 0.00 & 5.65 & 10.5 & 0.0 & 1.7 \\
\hline Navicula kuseliana Lange-Bertalot \& Rumrich & 8.0 & 0.00 & 2.67 & 14.3 & 0.00 & 5.80 & 5.3 & 0.0 & 0.5 \\
\hline $\begin{array}{l}\text { Navicula neomundana (Lange-Bertalot \& } \\
\text { Rumrich) Lange-Bertalot et al. }\end{array}$ & 42.0 & 0.00 & 7.00 & 11.4 & 0.00 & 1.75 & - & - & - \\
\hline Navicula notha Wallace & 94.0 & 0.00 & 19.73 & 54.3 & 0.00 & 18.20 & 31.6 & 0.0 & 7.1 \\
\hline Navicula rostellata Kützing & 4.0 & 0.00 & 0.40 & 25.7 & 0.00 & 12.92 & 21.1 & 0.0 & 7.9 \\
\hline Navicula symmetrica Patrick & 4.0 & 0.00 & 2.00 & - & - & - & - & - & - \\
\hline Nitzschia amphibia Grunow & - & - & - & 2.9 & 0.00 & 0.25 & 18.4 & 0.0 & 2.7 \\
\hline Nitzschia gracilis Hantzch & 4.0 & 0.00 & 2.59 & 20.0 & 0.00 & 2.07 & 5.3 & 0.0 & 0.4 \\
\hline Nitzschia palea (Kützing) Smith & 38.0 & 0.00 & 3.19 & 37.1 & 0.00 & 2.99 & 42.1 & 0.0 & 83.1 \\
\hline Nitzschia palea var. debilis (Kützing) Grunow & 28.0 & 0.00 & 2.25 & 8.6 & 0.00 & 1.50 & - & - & - \\
\hline **Nitzschia perminuta (Grunow) Peragallo & 8.0 & 0.00 & 2.04 & - & - & - & - & - & - \\
\hline *Nitzschia semirobusta Lange-Bertalot & 74.0 & 0.00 & 2.18 & 20.0 & 0.00 & 2.24 & - & - & - \\
\hline Nitzschia sp. 1 & - & - & - & 25.7 & 0.00 & 7.06 & 13.2 & 0.0 & 4.9 \\
\hline Nitzschia sp. 2 & 4.0 & 0.00 & 0.33 & 11.4 & 0.00 & 20.25 & - & - & - \\
\hline Nitzschia sp. 3 & - & - & - & - & - & - & 18.4 & 0.0 & 12.0 \\
\hline **Placoneis exigua (Gregory) Mereschkovsky & - & - & - & - & - & - & 5.7 & 0.0 & 3.7 \\
\hline $\begin{array}{l}\text { Planothidium rostratum (Østrup) Lange- } \\
\text { Bertalot }\end{array}$ & 6.0 & 0.00 & 0.44 & 42.9 & 0.00 & 11.46 & 5.3 & 0.0 & 1.9 \\
\hline $\begin{array}{l}\text { Pseudostaurosira brevistriata (Grunow) } \\
\text { Williams \& Round }\end{array}$ & 20.0 & 0.00 & 2.30 & 17.1 & 0.00 & 2.47 & - & - & - \\
\hline Pseudostaurosira sp. & 2.0 & 0.00 & 2.43 & 37.1 & 0.00 & 2.42 & 21.1 & 0.0 & 3.8 \\
\hline $\begin{array}{l}\text { Punctastriata lancettula (Schumann) Hamilton } \\
\text { \& Siver }\end{array}$ & 8.0 & 0.00 & 73.97 & 25.7 & 0.00 & 81.84 & 5.3 & 0.0 & 0.9 \\
\hline Rimoneis sp. & 6.0 & 0.00 & 2.63 & 20.0 & 0.00 & 6.94 & 5.3 & 0.0 & 2.1 \\
\hline Sellaphora nigri (De Notaris) Wetzel \& Ector & 4.0 & 0.00 & 2.46 & - & - & - & - & - & - \\
\hline${ }^{*}$ Sellaphora rostrata (Hustedt) Johansen & 14.0 & 0.00 & 1.47 & 17.1 & 0.00 & 5.43 & 2.6 & 0.0 & 0.2 \\
\hline $\begin{array}{l}\text { Sellaphora sassiana (Metzeltin \& Lange- } \\
\text { Bertalot) Wetzel }\end{array}$ & 40.0 & 0.00 & 3.58 & 14.3 & 0.00 & 9.48 & - & - & - \\
\hline Spicaticribra kingstonii Johansen et al. & 82.0 & 0.00 & 67.17 & 42.9 & 0.00 & 5.99 & 26.3 & 0.0 & 5.5 \\
\hline Staurosirella sp. & 4.0 & 0.00 & 0.40 & 34.3 & 0.00 & 3.92 & 13.2 & 0.0 & 3.7 \\
\hline Ulnaria acus (Kützing) Aboal & 8.0 & 0.00 & 0.65 & 17.1 & 0.00 & 2.67 & 18.4 & 0.0 & 3.9 \\
\hline
\end{tabular}


Table 4. Minimum (min) and maximum (max) relative abundances of diatoms from surface sediment and phytoplankton in summer and winter. - : taxon did not occur in sample; taxa in bold are detailed in the present study; *: first record for Brazil; **: first record for São Paulo State.

\begin{tabular}{|c|c|c|c|c|c|c|}
\hline \multirow[t]{2}{*}{ Species } & \multicolumn{2}{|c|}{ Surface sediment } & \multicolumn{2}{|c|}{$\begin{array}{c}\text { Summer } \\
\text { phytoplankton }\end{array}$} & \multicolumn{2}{|c|}{$\begin{array}{l}\text { Winter } \\
\text { phytoplankton }\end{array}$} \\
\hline & Min & Max & Min & Max & Min & Max \\
\hline Achnanthidium catenantum (Bily \& Marvan) Lange-Bertalot & 0.00 & 18.38 & 0.00 & 8.73 & 0.00 & 13.57 \\
\hline Achnanthidium exiguum (Grunow) Czarnecki & - & - & 0.00 & 26.80 & 0.00 & 25.58 \\
\hline *Achnanthidium jackii Rabenhorst & 0.00 & 5.66 & 0.00 & 24.69 & 0.00 & 10.04 \\
\hline *Achnanthidium lineare Smith & 0.00 & 6.47 & - & - & - & - \\
\hline $\begin{array}{l}\text { **Achnanthidium cf. macrocephalum (Hustedt) Round } \\
\text { \& Bukhtiyarova }\end{array}$ & - & - & 0.00 & 2.58 & 0.00 & 17.10 \\
\hline Achnanthidium minutissimum (Kützing) Czarnecki & 0.00 & 13.24 & 0.00 & 19.85 & 0.00 & 22.93 \\
\hline Achnanthidium tropicocatenatum Marquardt et al. & - & - & 0.00 & 2.95 & 0.00 & 44.04 \\
\hline Achnanthidium sp. & 0.00 & 6.28 & - & - & 0.00 & 0.86 \\
\hline Asterionella formosa Hassal & 0.00 & 2.01 & - & - & 0.00 & 2.33 \\
\hline Aulacoseira ambigua (Grunow) Simonsen & 0.21 & 63.56 & 0.00 & 26.93 & 0.00 & 44.42 \\
\hline Aulacoseira calypsi Tremarin et al. & 0.00 & 4.05 & 0.00 & 0.68 & 0.00 & 0.48 \\
\hline Aulacoseira granulata var. angustissima (Müller) Simonsen & 0.21 & 63.74 & 0.00 & 33.92 & 0.00 & 63.99 \\
\hline Aulacoseira granulata var. granulata (Ehrenberg) Simonsen & 0.00 & 51.64 & 0.00 & 19.90 & 0.00 & 88.36 \\
\hline Aulacoseira herzogii (Lemmermann) Simonsen & 0.00 & 2.47 & 0.00 & 0.94 & 0.00 & 1.22 \\
\hline Aulacoseira tenella (Nygaard) Simonsen & 0.00 & 54.72 & 0.00 & 38.40 & 0.00 & 83.95 \\
\hline Aulacoseria veraluciae Tremarin et al. & - & - & 0.00 & 2.07 & - & - \\
\hline Brachysira brebissonii Ross & - & - & - & - & 0.00 & 3.11 \\
\hline Brachysira microcephala (Grunow) Compère & 0.00 & 3.46 & 0.00 & 3.84 & 0.00 & 2.67 \\
\hline Brashysira sp. & 0.00 & 5.28 & - & - & - & - \\
\hline Cyclotella meneghiniana Kützing & 0.00 & 40.74 & 0.00 & 36.10 & 0.00 & 68.47 \\
\hline **Cymbella affinis var. neoprocera Silva & 0.00 & 6.91 & 0.00 & 0.45 & 0.00 & 0.50 \\
\hline $\begin{array}{l}\text { Cymbopleura naviculiformis (Auerswald ex Heiberg) } \\
\text { Krammer }\end{array}$ & 0.00 & 3.08 & 0.00 & 0.95 & 0.00 & 1.39 \\
\hline Diadesmis confervacea Kützing & 0.00 & 7.45 & 0.00 & 3.23 & 0.00 & 8.46 \\
\hline Discostella pseudostelligera (Hustedt) Houk \& Klee & - & - & 0.00 & 48.95 & 0.00 & 6.51 \\
\hline Discostella stelligera (Cleve \& Grunow) Houk \& Klee & 0.00 & 49.88 & 0.00 & 91.99 & 0.00 & 73.15 \\
\hline Encyonema silesiacum (Bleisch) Mann & 0.00 & 7.11 & 0.00 & 2.29 & 0.00 & 3.67 \\
\hline Encyonema sp. & 0.00 & 2.19 & 0.00 & 2.49 & 0.00 & 1.11 \\
\hline *Encyonopsis thienemannii (Hustedt) Krammer & - & - & 0.00 & 0.45 & 0.00 & 3.63 \\
\hline Eunotia botulitropica Wetzel \& Costa & 0.00 & 2.55 & 0.00 & 17.65 & 0.00 & 5.74 \\
\hline Eunotia desmogonioides Metzeltin \& Lange-Bertalot & - & - & 0.00 & 4.41 & - & - \\
\hline Eunotia intricans Lange-Bertalot \& Metzeltin & 0.00 & 28.64 & 0.00 & 1.50 & 0.00 & 3.33 \\
\hline Eunotia longicamelus Costa et al. & 0.00 & 2.49 & 0.00 & 18.78 & 0.00 & 3.67 \\
\hline Eunotia meridiana Metzeltin \& Lange-Bertalot & - & - & 0.00 & 2.29 & 0.00 & 0.50 \\
\hline Fragilaria aquaplus Lange-Bertalot \& Ulrich & 0.00 & 18.23 & 0.00 & 5.80 & 0.00 & 11.63 \\
\hline Fragilaria crotonensis Kitton & - & - & 0.00 & 2.75 & 0.00 & 0.37 \\
\hline *Fragilaria grunowii Lange-Bertalot \& Ulrich & 0.00 & 2.70 & 0.00 & 2.73 & 0.00 & 4.46 \\
\hline
\end{tabular}


Table 4 (continuation)

\begin{tabular}{|c|c|c|c|c|c|c|}
\hline \multirow[t]{2}{*}{ Species } & \multicolumn{2}{|c|}{ Surface sediment } & \multicolumn{2}{|c|}{$\begin{array}{c}\text { Summer } \\
\text { phytoplankton }\end{array}$} & \multicolumn{2}{|c|}{$\begin{array}{c}\text { Winter } \\
\text { phytoplankton }\end{array}$} \\
\hline & Min & Max & Min & $\operatorname{Max}$ & Min & Max \\
\hline Fragilaria longifusiformis (Hains \& Sebring) Siver et al. & 0.00 & 7.80 & 0.00 & 14.08 & 0.00 & 1.86 \\
\hline Fragilaria parva (Grunow) Tuji \& Williams & 0.00 & 8.96 & - & - & - & - \\
\hline Fragilaria spectra Almeida et al. & - & - & 0.00 & 46.38 & 0.00 & 0.96 \\
\hline $\begin{array}{l}\text { **Fragilaria tenera var. nanana (Lange-Bertalot) Lange- } \\
\text { Bertalot \& Ulrich }\end{array}$ & 0.00 & 3.93 & 0.00 & 3.62 & 0.00 & 30.42 \\
\hline Fragilaria sp. & 0.00 & 4.98 & 0.00 & 18.36 & 0.00 & 17.33 \\
\hline Geissleria punctifera (Hustedt) Metzeltin et al. & - & - & 0.00 & 1.17 & 0.00 & 2.99 \\
\hline Geissleria lateropunctata (Wallace) Potapova \& Winter & 0.00 & 3.27 & 0.00 & 1.88 & 0.00 & 7.65 \\
\hline Gomphonema hawaiense Reichardt & - & - & 0.00 & 1.33 & 0.00 & 2.00 \\
\hline Gomphonema lagenula Kützing & 0.00 & 1.96 & 0.00 & 11.03 & 0.00 & 1.48 \\
\hline **Gomphonema naviculoides Smith & 0.00 & 2.94 & 0.00 & 2.33 & 0.00 & 0.67 \\
\hline Gomphonema parvulum (Kützing) Kützing & 0.00 & 3.23 & - & - & - & - \\
\hline Hantzschia amphioxys (Ehrenberg) Grunow & 0.00 & 3.46 & - & - & - & - \\
\hline Humidophila contenta (Grunow) Lowe & 0.00 & 2.78 & 0.00 & 8.37 & 0.00 & 3.32 \\
\hline Navicula cryptocephala Kützing & 0.00 & 4.94 & 0.00 & 1.47 & 0.00 & 18.10 \\
\hline Navicula cryptotenella Lange-Bertalot & 0.00 & 5.65 & - & - & 0.00 & 4.85 \\
\hline Navicula kuseliana Lange-Bertalot \& Rumrich & 0.00 & 5.80 & 0.00 & 1.20 & 0.00 & 2.67 \\
\hline $\begin{array}{l}\text { Navicula neomundana (Lange-Bertalot \& Rumrich) Lange- } \\
\text { Bertalot et al. }\end{array}$ & 0.00 & 7.00 & 0.00 & 1.79 & 0.00 & 2.99 \\
\hline Navicula notha Wallace & 0.00 & 13.13 & 0.00 & 19.73 & 0.00 & 7.80 \\
\hline Navicula rostellata Kützing & 0.00 & 2.55 & 0.00 & 3.30 & 0.00 & 12.92 \\
\hline Navicula symmetrica Patrick & 0.00 & 2.00 & - & - & - & - \\
\hline Nitzschia amphibia Grunow & - & - & 0.00 & 2.24 & 0.00 & 2.74 \\
\hline Nitzschia gracilis Hantzch & - & - & 0.00 & 2.59 & - & - \\
\hline Nitzschia palea (Kützing) Smith & 0.00 & 3.19 & 0.00 & 83.06 & 0.00 & 5.50 \\
\hline Nitzschia palea var. debilis (Kützing) Grunow & - & - & 0.00 & 2.25 & 0.00 & 0.33 \\
\hline **Nitzschia perminuta (Grunow) Peragallo & 0.00 & 2.04 & - & - & - & - \\
\hline *Nitzschia semirobusta Lange-Bertalot & 0.00 & 2.18 & 0.00 & 2.24 & 0.00 & 1.00 \\
\hline Nitzschia sp. 1 & 0.00 & 7.06 & 0.00 & 1.23 & 0.00 & 1.11 \\
\hline Nitzschia sp. 2 & 0.00 & 20.25 & - & - & 0.00 & 3.32 \\
\hline Nitzschia sp. 3 & - & - & 0.00 & 4.04 & 0.00 & 12.00 \\
\hline **Placoneis exigua (Gregory) Mereschkovsky & 0.00 & 3.73 & 0.00 & 0.49 & - & - \\
\hline Planothidium rostratum (Østrup) Lange-Bertalot & 0.00 & 11.46 & 0.00 & 1.77 & 0.00 & 2.29 \\
\hline Pseudostaurosira brevistriata (Grunow) Williams \& Round & 0.00 & 2.47 & 0.00 & 0.72 & 0.00 & 0.97 \\
\hline Pseudostaurosira sp. & 0.00 & 2.97 & 0.00 & 3.77 & 0.00 & 1.46 \\
\hline Punctastriata lancettula (Schumann) Hamilton \& Siver & 0.00 & 81.84 & 0.00 & 23.49 & 0.00 & 15.87 \\
\hline Rimoneis sp. & 0.00 & 6.94 & 0.00 & 0.45 & 0.00 & 1.39 \\
\hline Sellaphora nigri (De Notaris) Wetzel \& Ector & 0.00 & 2.46 & - & - & - & - \\
\hline *Sellaphora rostrata (Hustedt) Johansen & 0.00 & 5.43 & 0.00 & 0.25 & 0.00 & 2.66 \\
\hline Sellaphora sassiana (Metzeltin \& Lange-Bertalot) Wetzel & 0.00 & 3.58 & 0.00 & 9.48 & 0.00 & 0.33 \\
\hline Spicaticribra kingstonii Johansen et al. & 0.00 & 67.17 & 0.00 & 12.56 & 0.00 & 3.23 \\
\hline Staurosirella sp. & 0.00 & 3.92 & 0.00 & 2.36 & 0.00 & 1.49 \\
\hline Ulnaria acus (Kützing) Aboal & - & - & 0.00 & 2.67 & 0.00 & 3.89 \\
\hline
\end{tabular}




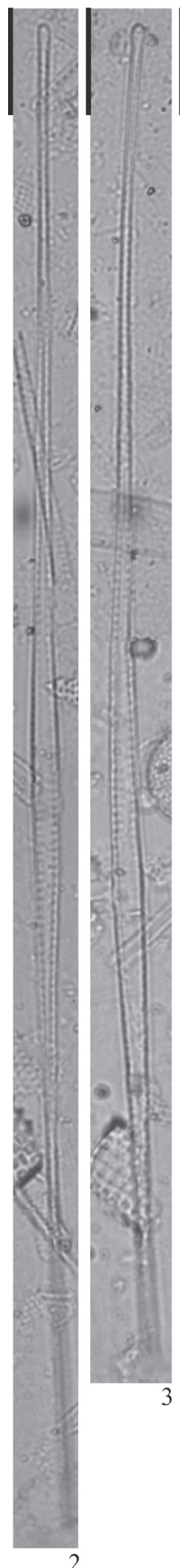

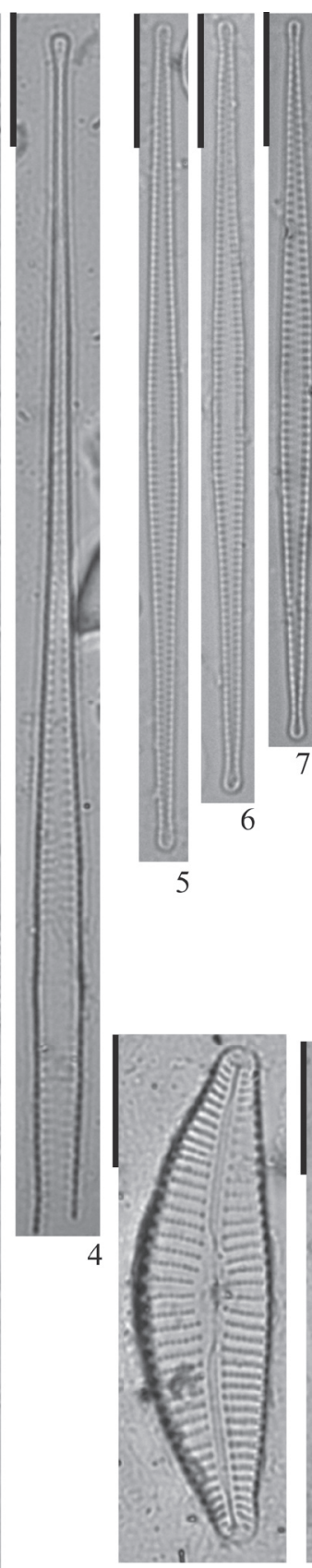

40
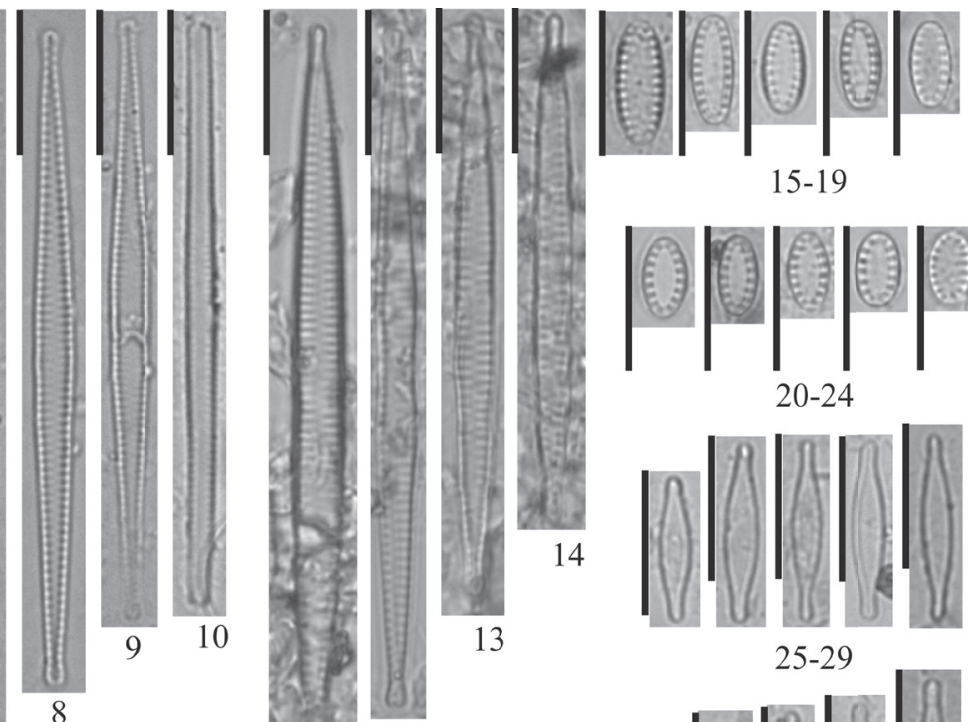

$15-19$
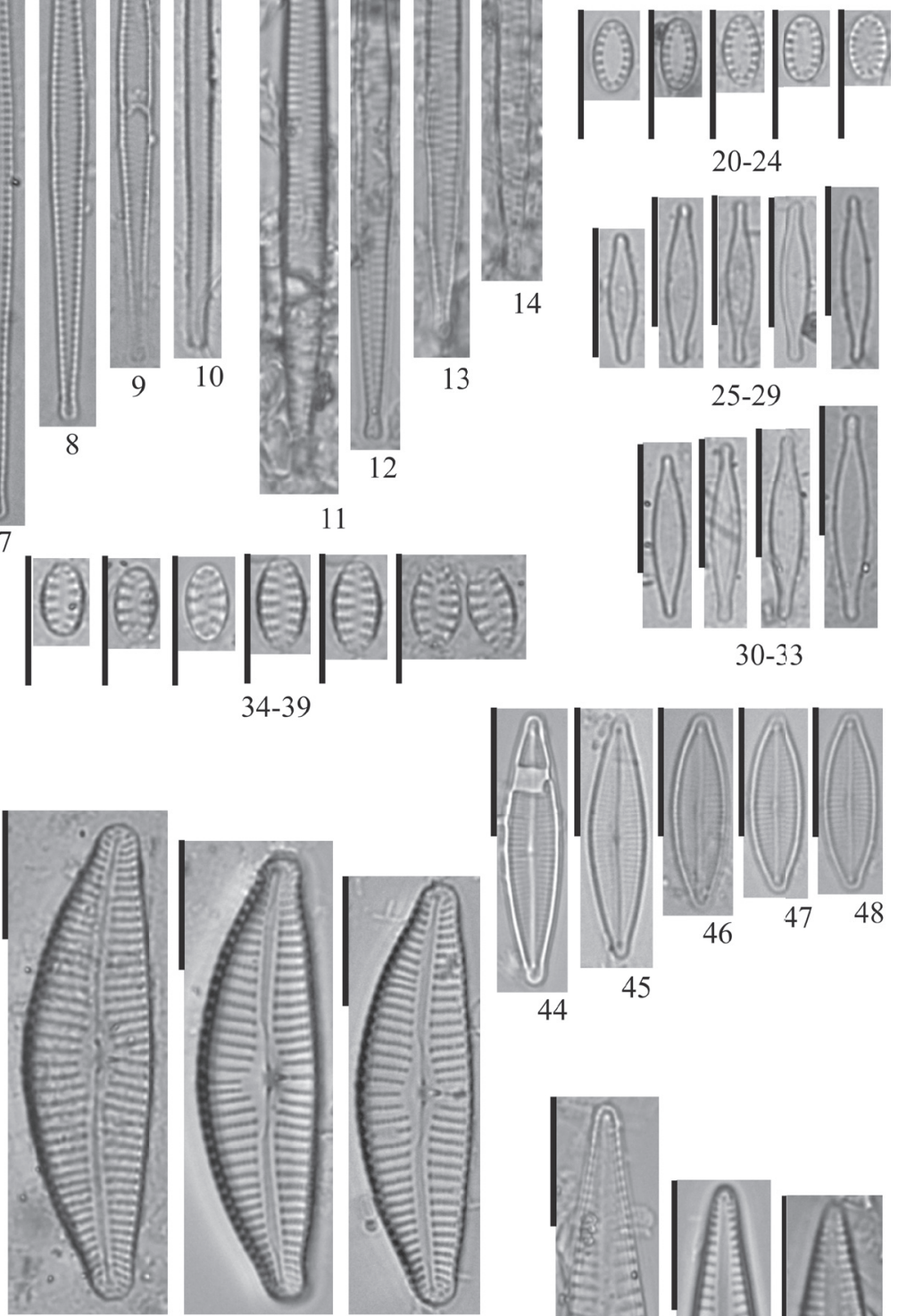

41
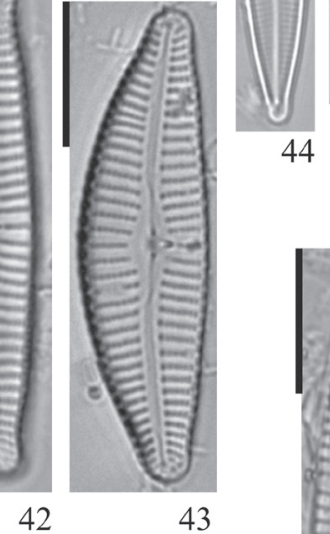

44
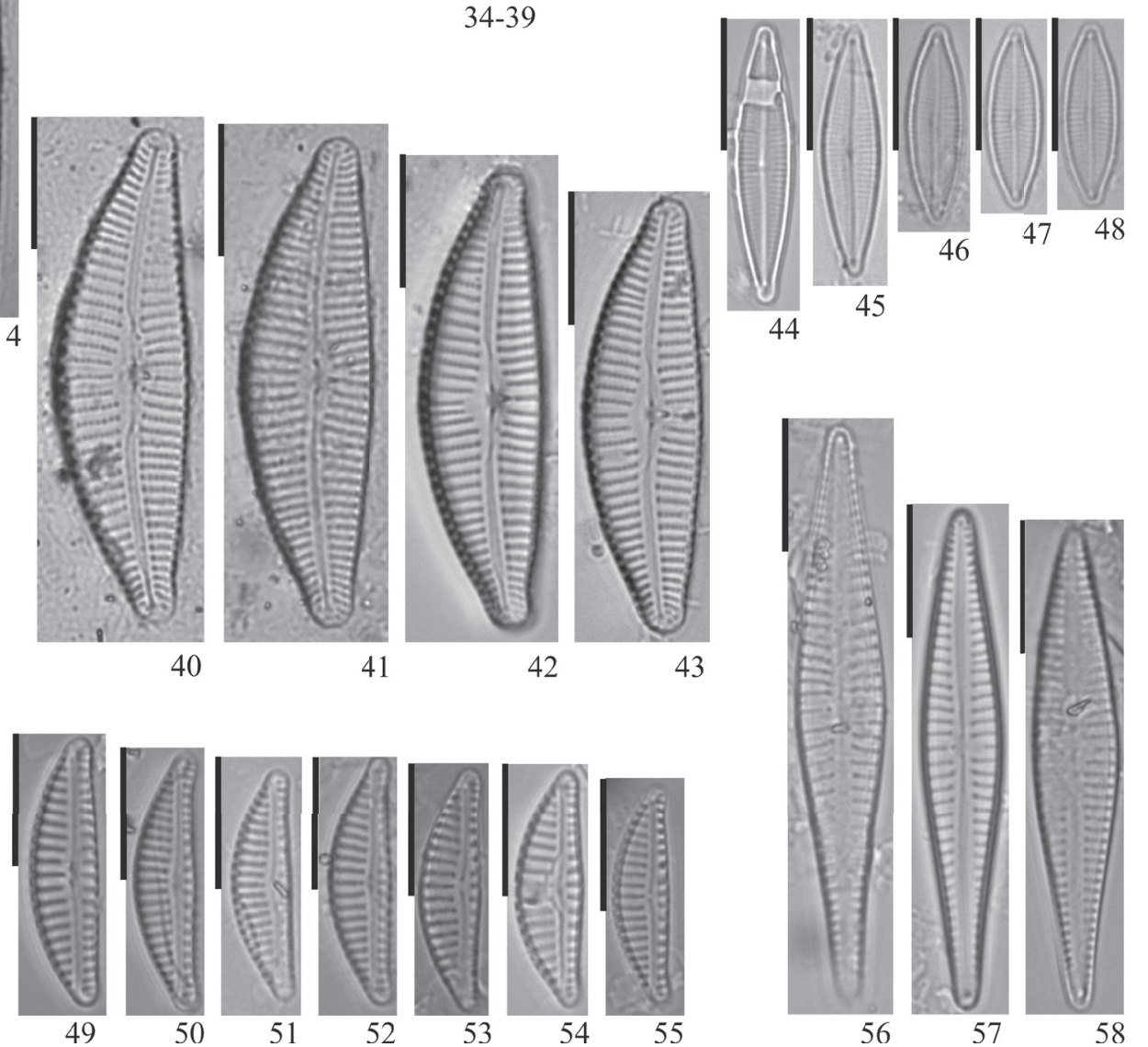

Figures 2-58. Surface sediment and planktonic diatoms. 2-4. Fragilaria grunowii Lange-Bertalot \& Ulrich. 5-10. Fragilaria tenera var. nanana (Lange-Bertalot) Lange-Bertalot \& Ulrich (Fig. 10. Lateral view). 11-14. Fragilaria sp. 15-24. Pseudostaurosira sp. 25-33. Rimoneis sp. 34-39. Staurosirella sp. 40-43. Cymbella affinis var. neoprocera Silva. 44-48. Encyonopsis thienemannii (Hustedt) Krammer. 49-55. Encyonema sp. 56-58. Gomphonema naviculoides Smith. Scale bar $=10 \mu \mathrm{m}$. 
Examined material: BRASIL. São PAULO: Sorocaba, Itupararanga, 29-VIII-2014, E. Bartozek \& D.C. Bicudo (surface sediment: SP469233, SP469234, SP469235, SP469236); Sorocaba, Itupararanga, 21-II-2014, E. Bartozek \& D.C. Bicudo (phytoplankton: SP469492, SP469493, SP469494, SP469495, SP469499, SP469500, SP469501); Votorantim, Santa Helena, 27-II-2014, E. Bartozek \& D.C. Bicudo (phytoplankton: SP469453, SP469454, SP469488); Ibiúna, Hedberg, 26-VIII-2014, E. Bartozek \& D.C. Bicudo (phytoplankton: SP469513).

**Fragilaria tenera var. nanana (Lange-Bertalot) Lange-Bertalot \& Ulrich, Lauterbornia 78, p. 7-8, pl. 2, fig. 1-6, pl. 4, fig. 1-6, 2014.

Figures 5-10

L: $34.1-66.1 \mu \mathrm{m}$; W: $2.4-3.0 \mu \mathrm{m}$; S: $17-20$ in 10 $\mu \mathrm{m}$.

Lange-Bertalot \& Ulrich (2014) provided new illustrations of $F$. tenera from the lectotype slide and presented three varieties: F. tenera (Smith) Lange-Bertalot var. tenera, $F$. tenera var. nanana (Lange-Bertalot) Lange-Bertalot \& Ulrich and $F$. tenera var. lemanensis Druart, Lavigne \& Robert. F. tenera var. nanana presents lanceolate valves, inflated and capitated ends and shorter measures (L: 50.0-70.0 $\mu \mathrm{m}$; W: $2.0 \mu \mathrm{m}$ ), while F. tenera var. tenera presents valves (sub)linear, mainly in the proximal region, slightly inflated ends, larger length (L: 60.0-120.0 $\mu \mathrm{m}$ ) and shorter width (W: 1.8-2.5 $\mu \mathrm{m}$ ). F. tenera var. lemanensis is characterized by longer $(\mathrm{L}$ : 97.0-102.0 $\mu \mathrm{m}$ ) and narrower valves (W: 1.5-1.7 $\mu \mathrm{m}$ ). This taxon was registered by Silva (2017) as Fragilaria tenera (Smith) Lange-Bertalot in the surface sediment and phytoplankton in seven of the studied reservoirs, with preferences for slightly acid to neutral waters $(\mathrm{pH}$ : 6.6-7.5) and low to moderate nutrients content (total nitrogen: 284.0-957.6 $\mu \mathrm{g} \mathrm{L}^{-1}$ and total phosphorus: 0.1-52.7 $\left.\mu \mathrm{g} \mathrm{L}^{-1}\right)$. In this study, it was observed in mesotrophic conditions in the surface sediment of one reservoir (Ipaneminha, maximum abundance: $3.9 \%$ ) and in phytoplankton of three meso- and hypereutrophic reservoirs (Itupararanga, Ipaneminha and Barra Bonita, maximum abundance: $30.4 \%$ ), but with higher frequency in mesotrophic conditions (25.7\%), corroborating previous information. This is the second register of the species in Brazil and the first published taxonomical register of this species.

Examined material: BRASIL, SÃo Paulo: Barra Bonita, Barra Bonita, 20-III-2014, E. Bartozek \& D.C. Bicudo (phytoplankton: SP469517); Sorocaba, Ipaneminha, 25-II-2014, E. Bartozek \& D.C. Bicudo (phytoplankton: SP469502, SP469503, SP469504); Sorocaba, Ipaneminha, 21-VIII-2014, E. Bartozek \& D.C. Bicudo (phytoplankton: SP469505, SP469506, SP469507); Sorocaba, Ipaneminha, 21-VIII-2014, E. Bartozek \& D.C. Bicudo (surface sediment: SP469237, SP469238, SP469239); Sorocaba, Itupararanga, 25-VIII-2017, E. Bartozek \& D.C. Bicudo (phytoplankton: SP469501); Sorocaba, Itupararanga, 25-VIII-2017, E. Bartozek \& D.C. Bicudo (surface sediment: SP469232).

\section{Fragilaria sp.}

Figures 11-14

Valves linear with weakly convex margin; ends subcapitate; axial area narrow and linear; central area bilaterally swelling and with "ghost" striae; striae parallel. L: 27.0-59.0 $\mu \mathrm{m}$; W: 2.0-4.0 $\mu \mathrm{m}$; S: 14-16 in $10 \mu \mathrm{m}$.

This taxon resembles F. parva (Grunow) Tuji \& Williams, which however presents more pronounced swellings and capitate ends (Tuji \& Williams 2008) than Fragilaria sp. This species was recorded in the surface sediment of one mesotrophic reservoir (Tatu, maximum abundance: $5.0 \%$ ), and in phytoplankton of four reservoirs ranging from oligo- to mesotrophic conditions (Atibainha, Paiva Castro, Cachoeira and Tatu, maximum abundance: $18.3 \%$ ). The highest frequency in samples was in oligotrophic conditions $(22.0 \%)$.

Examined material: BRASIL. São PAUlo: Limeira, Tatu, 18-IV-2013, S. Zorzal-Almeida \& D.C. Bicudo (phytoplankton: SP469289, SP469290, SP469291); Limeira, Tatu, 18-VII-2013, S. ZorzalAlmeida \& D.C. Bicudo (phytoplankton: SP469311, SP469312, SP469313; surface sediment: SP469269); Mairiporã, Paiva Castro, 18-I-2013, S. ZorzalAlmeida \& D.C. Bicudo (phytoplankton: SP469281, SP469282, SP469283, SP469284); Mairiporã, Paiva Castro, 19-VII-2013, S. Zorzal-Almeida \& D.C. Bicudo (phytoplankton: SP469303, SP469304, SP469305, SP469306); Nazaré Paulista, Atibainha, 24-I-2013, S. Zorzal-Almeida \& D.C. Bicudo (phytoplankton: SP469275, SP469278); Piracaia, Cachoeira, 26-VII-2013, S. Zorzal-Almeida \& D.C. Bicudo (phytoplankton: SP469292).

Pseudostaurosira Williams \& Round

\section{Pseudostaurosira sp.}

Figures 15-24 
Valves elliptical to elliptical-lanceolate, axial area wide, following the valve shape; striae short, parallel in the center becoming slightly radiate toward the ends. L: $5.3-8.9 \mu \mathrm{m}$; W: $2.5-3.3 \mu \mathrm{m}$; S: $16-17$ in $10 \mu \mathrm{m}$.

This taxon was identified as belonging to the genus Pseudostaurosira due to the uniseriate striae and wide axial area. Other features, such as presence of spines and absent or reduced apical pore fields are only visible in SEM (Morales 2001). Pseustaurosira sp. resembles Fragilaria canariensis Lange-Bertalot due to their similar valve shape. Neverthless, the second species is shorter (L: 4.0-7.0 $\mu \mathrm{m}$ ), wider (W: 3.0-4.0 $\mu \mathrm{m}$ ) and exhibits smaller striae density (S: $12-15$ in $10 \mu \mathrm{m}$ ) than Pseustaurosira sp (Lange-Bertalot 1993). It can be also misidentified as Staurosirella sp. (see taxon bellow), however, Pseudostaurosira sp. presents shorter striae and wider axial area. This is probably a new diatom species for science, since its general features differs from others species available in the literature. The species was documented in the surface sediment of four reservoirs with trophic state ranging from oligo- to eutrophic (Santa Helena, Itupararanga, Ipaneminha, and Hedberg, maximum abundance: $3.0 \%$ ), and in the phytoplankton of four reservoirs ranging from oligo- to eutrophic conditions (Santa Helena, Itupararanga, Ipaneminha and Hedberg, maximum abundance: $3.8 \%$ ). The highest frequency occurred in mesotrophic conditions $(37.1 \%)$.

Examined material: BRASIL. São Paulo: Iperó, Hedberg, 12-III-2014, E. Bartozek \& D.C. Bicudo (phytoplankton: SP469508, SP469509, SP469510); Iperó, Hedberg, 26-VIII-2014, E. Bartozek \& D.C. Bicudo (phytoplankton: SP469511, SP469512, SP469513; surface sediment: SP469240, SP469241, SP469242); Sorocaba, Ipaneminha, 21-VIII-2014, E. Bartozek \& D.C. Bicudo (phytoplankton: SP469505, SP469507; surface sediment: SP469238, SP469239); Sorocaba, Itupararanga, 25-VIII-2014, E. Bartozek \& D.C. Bicudo (phytoplankton: SP469500; surface sediment: SP469235, SP469236); Sorocaba, Itupararanga, 29-VIII-2014, E. Bartozek \& D.C. Bicudo (phytoplankton: SP469499; surface sediment: SP469233, SP469234); Votarantim, Santa Helena, 27-II-2014, E. Bartozek \& D.C. Bicudo (phytoplankton: SP469453); Votarantim, Santa Helena, 21-VIII-2014, E. Bartozek \& D.C. Bicudo (surface sediment: SP469230, SP469231).

\section{Rimoneis Garcia}

\section{Rimoneis sp.}

Figures 25-33
Valves linear-lanceolate and hyaline; valve face with wide axial area occupying almost all the valve face; rounded ends. L: 9.2-16.1 $\mu \mathrm{m}$; W: 1.5-3.0 $\mu \mathrm{m}$; inconspicuous striae.

The species resembles Rimoneis inanis Garcia in relation to the valve shape. However, $R$. inanis presents larger valve length (L: 17.0-25.0 $\mu \mathrm{m}$, Garcia 2010). This author points out that $R$. inanis presented restricted distribution to freshwater/brackish water of the Lagoa dos Patos lagoon. Rimoneis sp. is described as a new species in the revision carried out by (Silva 2017) and mainly associated to low nutrient content environments. In this study, it occurred in the surface sediment of five reservoirs ranging from oligo- to mesotrophic conditions (Santa Helena, Atibainha, Cachoeira, Paiva Castro and Itupararanga, maximum abundance: $7.0 \%$ ) and in the phytoplankton of mesotrophic reservoir Itupararanga (maximum abundance: $1.4 \%$ ). Nevertheless, highest frequency in samples was observed in mesotrophic conditions $(20.0 \%)$.

Examined material: BRASIL. S̃̃o PAULO: Mairiporã, Paiva Castro, 19-VII-2013, S. Zorzal-Almeida \& D.C. Bicudo (surface sediment: SP469262); Nazaré Paulista, Atibainha, 25-VII-2013, S. Zorzal-Almeida \& D.C. Bicudo (surface sediment: SP469253); Piracaia, Cachoeira, 26-VII-2013, S. Zorzal-Almeida \& D.C. Bicudo (surface sediment: SP469250); Sorocaba, Itupararanga, 21-II-2014, E. Bartozek \& D.C. Bicudo (phytoplankton: SP469493); Sorocaba, Itupararanga, 25-VIII-2014, E. Bartozek \& D.C. Bicudo (phytoplankton: SP469500, SP469501; surface sediment: SP469235, SP469236); Sorocaba, Itupararanga, 29-VIII-2014, E. Bartozek \& D.C. Bicudo (phytoplankton: SP469498; surface sediment: SP469233, SP469234); Votorantim, Santa Helena, 21-VIII-2014, E. Bartozek \& D.C. Bicudo (surface sediment: SP469231).

Staurosirella Williams \& Round

\section{Staurosirella sp.}

Figures 34-39

Valves oval to elliptic; ends rounded; axial area linear and narrow; striae alternated and slightly radiate; central area absent. L: 4.7-7.3 $\mu \mathrm{m}$; W: 3.4-4.3 $\mu \mathrm{m}$; S: 12 in $10 \mu \mathrm{m}$.

This taxon resembles Staurosira altiplanensis Lange-Bertalot \& Rumrich due to the overlapping measures (L: $4.5 \mu \mathrm{m}$; W: 2.8-3.6 $\mu \mathrm{m}$ ) and similar 
valve outline. However, S. altiplanensis presents valves strictly elliptical and was described for the high altitude of the Altiplano of South America (Lange-Bertalot 2000). Furthermore, Staurosirella sp. was identified as Staurosirella pinnata (Ehrenberg) Williams \& Round by Ribeiro et al. (2008) and Nardelli et al. (2014). However, Morales et al. (2013) demonstrated that $S$. pinnata type material, originally described as Fragilaria pinnata Ehrenberg, represents a species of the genus Denticula Kützing. Therefore, for these authors, a prior careful taxonomical and ecological analysis of this species is necessary. It was presently found in the surface sediment of four reservoirs with trophic states from oligo- to eutrophic conditions (Santa Helena, Itupararanga, Ipaneminha and Hedberg, maximum abundance: $4.0 \%$ ), and in the phytoplankton of three reservoirs ranging from oligoto eutrophic conditions (Santa Helena, Itupararanga and Hedberg, maximum abundance: $2.3 \%$ ), and with higher frequency in mesotrophic waters (34.3\%).

Examined material: BRASIL. São PAULO: Iperó, Hedberg, 12-III-2014, E. Bartozek \& D.C. Bicudo (phytoplankton: SP469508, SP469509, SP469510); Iperó, Hedberg, 26-VIII-2014, E. Bartozek \& D.C. Bicudo (phytoplankton: SP469511, SP469512; surface sediment: SP469240, SP469241, SP469242); Sorocaba, Ipaneminha, 21-VIII-2014, E. Bartozek \& D.C. Bicudo (surface sediment: SP469237, SP469238); Sorocaba, Itupararanga, 21-II-2014, E. Bartozek \& D.C. Bicudo (phytoplankton: SP469496); Sorocaba, Itupararanga, 25-VIII-2014, E. Bartozek \& D.C. Bicudo (phytoplankton: SP469500; surface sediment: SP469235, SP469236); Sorocaba, Itupararanga, 29-VIII-2014, E. Bartozek \& D.C. Bicudo (surface sediment: SP469234); Votorantim, Santa Helena, 27-II-2014, E. Bartozek \& D.C. Bicudo (phytoplankton: SP469454, SP469488); Votorantim, Santa Helena, 21-VIII-2014, E. Bartozek \& D.C. Bicudo (surface sediment: SP469229, SP469231).

Cymbellaceae Greville

\section{Cymbella Agardh}

**Cymbella affinis var. neoprocera Silva, PhytoKeys 53, p. 10-11, figs. 22-28, 2015.

Figures 40-43

L: 34.5-39.4 $\mu \mathrm{m}$; W: 9.4-11.3 $\mu \mathrm{m}$; $\mathrm{S}: 9-11$ in $10 \mu \mathrm{m}$.

C. affinis var. neoprocera is the synonymous of Cymbella excisa var. procera Krammer. This taxon can be compared to Cymbella tropica Krammer, however, our species exhibits more slightly protracted ends than C. tropica (Silva et al. 2015). This taxon was recorded in the Paraná state by Ludwig et al. (2005) as Cymbella affinis Kützing. According to Krammer (2002), this taxon is usually abundant in eutrophic waters. In this study, it occurred in oligo- to eutrophic conditions in three reservoirs (Santa Helena, Ipaneminha and Hedberg) for both surface sediment and phytoplankton (maximum abundance: $7.0 \%$ and $0.5 \%$, respectively), with higher frequency in eutrophic waters $(10.5 \%)$, corroborating previous finding.

Examined material: BRASIL. São PAUlo: Iperó, Hedberg, 12-III-2017, E. Bartozek \& D.C. Bicudo (phytoplankton: SP469508); Iperó, Hedberg, 26-VIII-2017, E. Bartozek \& D.C. Bicudo (phytoplankton: SP469512, SP469513; surface sediment: SP469240, SP469241); Sorocaba, Ipaneminha, 21-VIII-2014, E. Bartozek \& D.C. Bicudo (phytoplankton: SP469505, SP469506, SP469507; surface sediment: SP469237, SP469238); Votorantim, Santa Helena, 27-II-2014, E. Bartozek \& D.C. Bicudo (phytoplankton: SP469454, SP469488); Votorantim, Santa Helena, 27-II-2014, E. Bartozek \& D.C. Bicudo (surface sediment: SP469231).

\section{Encyonema Kützing}

\section{Encyonema sp.}

Figures 49-55

Valve strongly dorsiventral, semi-lanceolate, with dorsal margin strongly convex and ventral margin straight to slightly concave and expanded in the middle portion; ends ventrally bent; axial area straight and linear; raphe straight, external ends deflected to the ventral side and proximal ends deflected to the dorsal side. L: $11.0-22.2 \mu \mathrm{m}$; W: $4.0-5.8 \mu \mathrm{m}$; S: $10-13$ in $10 \mu \mathrm{m}$.

This taxon resembles E. simile Krammer in valve shape and measures (L: 16.0-25.5 $\mu \mathrm{m}$; W: 5.1-6.0 $\mu \mathrm{m}$; S: 10-13 in $10 \mu \mathrm{m}$; Krammer 1997), however Encyonema sp. presents thinner and slightly more capitate ends. Further analyses (e.g., MEV) are required to confirm if this taxon is a new species. In this study, it was found in the surface sediment of three oligotrophic reservoirs (Atibainha, Cachoeira and Paiva Castro, maximum abundance: $2.2 \%$ ), and in the phytoplankton of three reservoirs ranging from oligo- to mesotrophic conditions (Cachoeira, Paiva Castro and Tatu, maximum abundance: 4.8), 
and presented higher frequency of occurrence in oligotrophic conditions $(26.0 \%)$.

Examined material: BRASIL. São PAUlo: Limeira, Tatu, 18-IV-2013, S. Zorzal-Almeida \& D.C. Bicudo (phytoplankton: SP469290); Mairiporã, Paiva Castro, 19-VII-2013, S. Zorzal-Almeida \& D.C. Bicudo (phytoplankton: SP469304; surface sediment: SP469259, SP469260, SP469261, SP469262); Nazaré Paulista, Atibainha, 25-VII-2013, S. Zorzal-Almeida \& D.C. Bicudo (surface sediment: SP469253, SP469254); Piracaia, Cachoeira, 21-II-2013, S. Zorzal-Almeida \& D.C. Bicudo (phytoplankton: SP469271, SP469272, SP469273); Piracaia, Cachoeira, 26-VII-2013, S. Zorzal-Almeida \& D.C. Bicudo (phytoplankton: SP469292; surface sediment: SP469248; SP469249, SP469250).

\section{Encyonopsis Krammer}

*Encyonopsis thienemannii (Hustedt) Krammer, Bibliotheca Diatomologica 37, p. 106, pl. 149, fig. 28-33, 1997.

Figures 44-48

L: 13.2-21.1; W: 4.0-4.7; S: 25-29 in $10 \mu \mathrm{m}$.

E. thienemannii resembles E. minuta Krammer \& Reichardt in relation to valve outline. However, the second species shows more detached apices, narrower width (W: 2.8-3.5 $\mu \mathrm{m}$ ) and lower striae density (S: 24-25 in $10 \mu \mathrm{m}$; Krammer 1997) than E. thienemannii. According to Krammer (1997) this species is found in springs and falls. In this study, it was recorded exclusively in the phytoplankton of two oligo- and mesotrophic reservoirs (Santa Helena and Itupararanga, maximum abundance: $3.6 \%$ ), with higher frequency in mesotrophic conditions (5.7\%).

Examined material: BRASIL. São PaUlo: Sorocaba, Itupararanga, 21-II-2014, E. Bartozek \& D.C. Bicudo (phytoplankton: SP469493); Votorantim, Santa Helena, 21-VIII-2014, E. Bartozek \& D.C. Bicudo (phytoplankton: SP469489, SP469490, SP469491).

Gomphonema Ehrenberg

**Gomphonema naviculoides Smith, A synopsis of the British Diatomaceae, v. 2 pp. [i-vi] - xxix, 1-107, pls. 32-60, 61-62, A-E, 1856.

Figures 56-58

L: $22.0-55.0 \mu \mathrm{m} ; \mathrm{W}: 5.0-8.2 \mu \mathrm{m}$; $\mathrm{S}: 10-15$ in $10 \mu \mathrm{m}$.
Gomphonema naviculoides belongs to Gomphonema gracile complex sensu Grunow et sensu auct, and according to Reichardt (2015) both species can be easily mistaken. However, this author highlights that G. naviculoides presents valves lanceolate to rhombiclanceolate and almost naviculoid-symmetrical, while G. gracile presents valves lanceolate, gomphonemoidclavate shaped with bluntly rounded poles. In this study, the species occurred in the surface sediment of two oligo- and mesotrophic reservoirs (Paiva Castro and Tatu, maximum abundance: $3.0 \%$ ), and in the phytoplankton of three oligo- and mesotrophic reservoirs (Cachoeira, Paiva Castro and Tatu, maximum abundance: $2.3 \%$ ), and with higher frequency in mesotrophic conditions (14.3\%).

Examined material: BRASIL. São PaUlo: Limeira, Tatu, 18-IV-2013, S. Zorzal-Almeida \& D.C. Bicudo (phytoplankton: SP469289, SP469290, SP469291); Limeira, Tatu, 18-VII-2013, S. Zorzal-Almeida \& D.C. Bicudo (phytoplankton: SP469313; surface sediment: SP469267, SP469269); Mairiporã, Paiva Castro, 19-VII-2013, S. Zorzal-Almeida \& D.C. Bicudo (phytoplankton: SP469303; surface sediment: SP469260); Piracaia, Cachoeira, 21-II-2013, S. ZorzalAlmeida \& D.C. Bicudo (phytoplankton: SP469271); Piracaia, Cachoeira, 26-VII-2013, S. Zorzal-Almeida \& D.C. Bicudo (phytoplankton: SP469295).

\section{Placoneis Mereschkovsky}

**Placoneis exigua (Gregory) Mereschkovsky, Beihefte zum Botanischen Centralblatt 15(1),p. 1-30, pl.1, 1903.

Figures 59-61

L: 24.2-33.8 $\mu \mathrm{m}$; W: 9.7-11.2 $\mu \mathrm{m}$; S: 10-14 in $10 \mu \mathrm{m}$. This taxon resembles Placoneis constans (Husted) Cox var. symmetrica (Hustedt) Kobayasi due to their lanceolate valves and rostrate ends. However, the second species is smaller (L: 19.3-25.6 $\mu \mathrm{m}, \mathrm{W}:$ 8.1-9.3 $\mu \mathrm{m})$ than $P$. exigua and presents a central area with a longer striae surrounded by shorter striae (Marquardt \& Bicudo 2014). Placoneis exigua is reported as an indicator of eutrophic conditions (Van Dam et al. 1994, Besse-Lototskaya et al. 2011). In this study it occurred in surface sediment of eu- and hypereutrophic reservoirs (Hedberg and Barra Bonita, maximum abundance: $3.7 \%$ ), and in the phytoplankton of eutrophic reservoir Hedberg (maximum abundance: $0.5 \%$ ), with $5.7 \%$ frequency in samples, corroborating previous finding.

Examined material: BRASIL. S̃̃o Paulo: Barra Bonita, Barra Bonita, 29-VII-2014, E. Bartozek \& 
D.C. Bicudo (surface sediment: SP469245); Iperó, Hedberg, 12-III-2014, E. Bartozek \& D.C. Bicudo (phytoplankton: SP469508, SP469510); Iperó, Hedberg, 26-VIII-2014, E. Bartozek \& D.C. Bicudo (surface sediment: SP469240).

Achnanthidiaceae Mann

\section{Achnanthidium Kützing}

*Achnanthidium jackii Rabenhorst, Die Algen Europas, Fortsetzung der Algen Sachsens, resp. Mittel-Europas. Decades I-CIX, numbers 1-1600 (or 1001-2600), 1861.

Figures 62-67
L: 7.3-15.0 $\mu \mathrm{m}$; W: 3.4-3.7 $\mu \mathrm{m}$; inconspicuous striae.

This taxon can be misidentified as A. minutissimum (Kützing) Czarnecki since they have similar valve shape. However, A. jackii is usually wider than $A$. minutissimum (W: 2.5-3.0 $\mu \mathrm{m}$ ) and doesn't exhibits a shortened striae on one side of the central area as in the second species (see figure 62, Wojtal et al. 2011). It is mostly found in neutral environments with low organic matter concentrations (Van Dam et al. 1994, Wojtal et al. 2011). In this study, it occurred in the surface sediment of seven reservoirs ranging from oligo- to eutrophic conditions (Atibainha, Cachoeira, Itupararanga, Paiva Castro, Tatu, Hedberg and

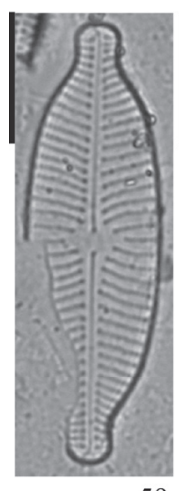

59

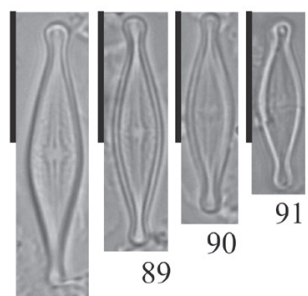

88

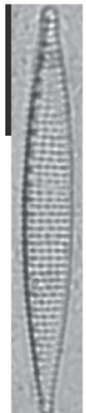

102

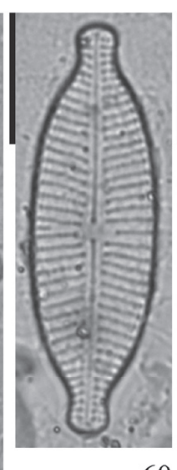

60

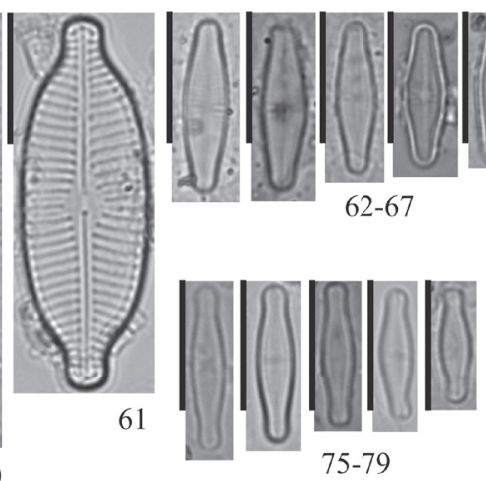

75-79

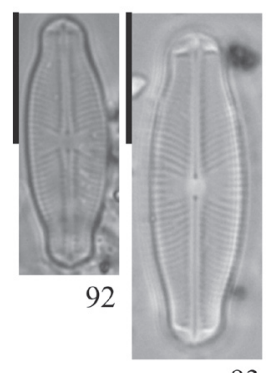

93

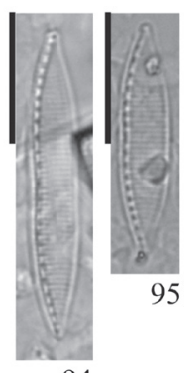

94
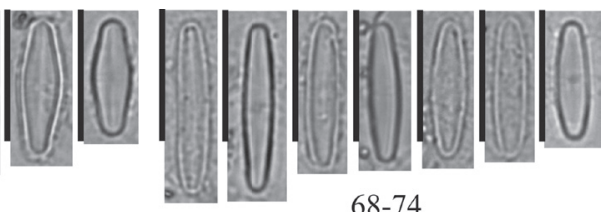

68-74
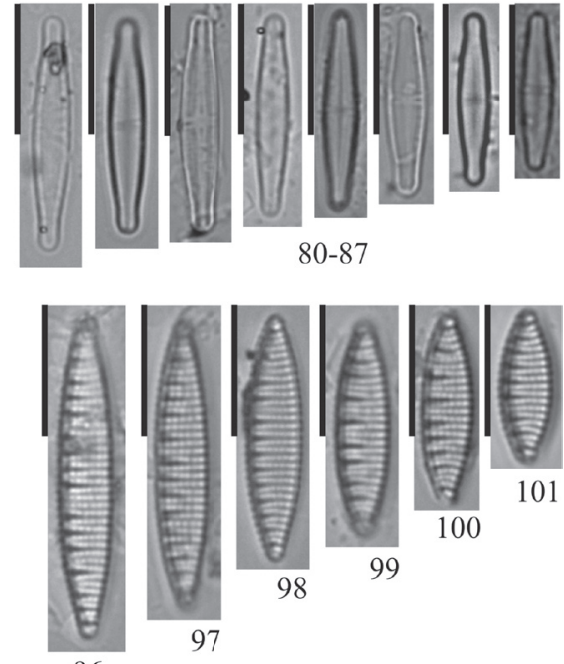

Figures 59-112. Surface sediment and planktonic diatoms. 59-61. Placoneis exigua (Gregory) Mereschkovsky. 62-67. Achnanthidium jackii Rabenhorst. 68-74. Achnanthidium lineare Smith. 75-79. Achnanthidium cf. macrocephalum (Hustedt) Round \& Bukhtiyarova. 80-87. Achnanthidium sp. 1. 88-91. Brachysira sp. 92-93. Sellaphora rostrata (Hustedt) Johansen. 94-95. Nitzschia perminuta (Grunow) Peragallo. 96-101. Nitzschia semirobusta Lange-Bertalot. 102-109. Nitzschia sp. 1. 110-112. Nitzschia sp. 2. Scale bar $=10 \mu \mathrm{m}$. 
Salto Grande, maximum abundance: $5.6 \%$ ), and in the phytoplankton of six reservoirs ranging from oligo- to eutrophic conditions (Atibainha, Cachoeira, Santa Helena, Paiva Castro, Tatu and Salto Grande, maximum abundance: $24.7 \%$ ), showing higher frequency in oligotrophic samples $(76.0 \%)$.

Examined material: BRASIL. São PAULO: Americana, Salto Grande, 9-III-2013, S. Zorzal-Almeida \& D.C. Bicudo (phytoplankton: SP469285, SP469286, SP469287, SP469288); Americana, Salto Grande, 20-IX-2013, S. Zorzal-Almeida \& D.C. Bicudo (phytoplankton: SP469307, SP469308, SP469309, SP469310; surface sediment: SP469264); Iperó, Hedberg, 26-VIII-2014, E. Bartozek \& D.C. Bicudo (phytoplankton: SP469512, SP469513; surface sediment: SP469240, SP469241, SP469242); Limeira, Tatu, 18-IV-2013, S. Zorzal-Almeida \& D.C. Bicudo (phytoplankton: SP469289, SP469290, SP469291); Limeira, Tatu, 18-VII-2013, S. ZorzalAlmeida \& D.C. Bicudo (phytoplankton: SP469311, SP469312, SP469313; surface sediment: SP469267); Mairiporã, Paiva Castro, 18-I-2013, S. ZorzalAlmeida \& D.C. Bicudo (phytoplankton: SP469281, SP469282, SP469283, SP469284); Mairiporã, Paiva Castro, 19-VII-2013, S. Zorzal-Almeida \& D.C. Bicudo (phytoplankton: SP469303, SP469304, SP469306; surface sediment: SP469259, SP469260, SP469261, SP469262); Nazaré Paulsita, Atibainha, 24-I-2013, S. Zorzal-Almeida \& D.C. Bicudo (phytoplankton: SP469275, SP469276, SP469277, SP469278, SP469279, SP469280); Nazaré Paulsita, Atibainha, 25-VII-2013, S. Zorzal-Almeida \& D.C. Bicudo (phytoplankton: SP469297, SP469298; surface sediment: SP469253, SP469254, SP469255, SP469256, SP469257, SP469258); Piracaia, Cachoeira, 21-II-2013, S. Zorzal-Almeida \& D.C. Bicudo (phytoplankton: SP469270, SP469271, SP469272, SP469273, SP469274); Piracaia, Cachoeira, 26-VII-2013, S. Zorzal-Almeida \& D.C. Bicudo (phytoplankton: SP469292, SP469293, SP469295; surface sediment: SP469248, SP469249, SP469251, SP469252); Sorocaba, Itupararanga, 25-VIII-2014, E. Bartozek \& D.C. Bicudo (surface sediment: SP469232, SP469235, SP469236); Votorantim, Santa Helena, 27-II-2014, E. Bartozek \& D.C. Bicudo (phytoplankton: SP469488); Votorantim, Santa Helena, 21-VIII-2014, E. Bartozek \& D.C. Bicudo (phytoplankton: SP469489, SP469490).

* Achnanthidium lineare Smith, Annals and Magazine of Natural History, series 2 15, pl. 1, fig. 1-9, 1855. Figures 68-74
L: 9.0-13.4 $\mu \mathrm{m}$; W: 2.0-2.8 $\mu \mathrm{m}$; inconspicuous striae.

Achnanthidium lineare presents linear (almost parallel margins) to narrowly lanceolate valves, while in A. minutissimum they are lanceolate to ellipticlanceolate (Wojtal et al. 2011). Furthermore, the first taxon presents rounded to rostrate apices and the second presents rostrate to subcapitate apices (Van de Vijver et al. 2011). This species is found mostly in circumneutral waters (Van Dam et al. 1994). In the present study, it was found in surface sediment of four reservoirs ranging from oligo- to mesotrophic conditions (Ipaneminha, Itupararanga, Santa Helena and Tatu, maximum abundance: $6.5 \%$ ), with frequency of $14.3 \%$ in mesotrophic samples.

Examined material: BRASIL. SÃo PAUlo: Limeira, Tatu, 18-VII-2013, S. Zorzal-Almeida \& D.C. Bicudo (surface sediment: SP469268, SP469269). Sorocaba, Ipaneminha, 21-VIII-2014, E. Bartozek \& D.C. Bicudo (surface sediment: SP469237); Sorocaba, Itupararanga, 25-VIII-2014, E. Bartozek \& D.C. Bicudo (surface sediment: SP469232); Sorocaba, Itupararanga, 29-VIII-2014, E. Bartozek \& D.C. Bicudo (surface sediment: SP469233, SP469234); Votorantim, Santa Helena, 21-VIII-2014, E. Bartozek \& D.C. Bicudo (surface sediment: SP469229).

**Achnanthidium cf. macrocephalum (Hustedt) Round \& Bukhtiyarova, Diatom Research 11(2), p. $349,1996$.

Figures 75-79

L: 9.0-14.0 $\mu \mathrm{m}$; W: 2.0-3.0 $\mu \mathrm{m}$; inconspicuous striae.

This taxon was identified as $A$. cf. macrocephalum because our specimens showed ends slightly less rounded than those presented in Simonsen (1987). Individuals similar to ours were recorded by Taylor et al. (2007) and Marra et al. (2016) as A. macrocephalum. Our taxon can be distinguished from A. minutissimum (Kützing) Czarnecki mainly due to its capitate ends (Potapova \& Hamilton 2007). According to these authors, $A$. macrocephalum is an alkaliphilous species, however, they recorded other more capitate specimens in slightly acid and nutrient-poor waters of North America. Marra et al. (2016) recorded this species among the ten most frequent species in a mesotrophic subtropical reservoir. In this study, it was found in phytoplankton of five reservoirs ranging from oligo- to eutrophic conditions (Atibainha, Cachoeira, Paiva Castro, Tatu and Salto Grande, maximum 
abundance: $17.1 \%$ ), and with higher frequency in oligotrophic conditions (30.0\%).

Examined material: BRASIL. São PAULO: Americana, Salto Grande, 9-III-2013, S. Zorzal-Almeida \& D.C. Bicudo (phytoplankton: SP469287); Americana, Salto Grande, 20-IX-2013, S. Zorzal-Almeida \& D.C. Bicudo (phytoplankton: SP469307, SP469308, SP469309, SP469310); Limeira, Tatu, 18-IV-2013, S. Zorzal-Almeida \& D.C. Bicudo (phytoplankton: SP469289, SP469290, SP469291); Limeira, Tatu, 18-VII-2013, S. Zorzal-Almeida \& D.C. Bicudo (phytoplankton: SP469311, SP469312, SP469313); Mairiporã, Paiva Castro, 18-I-2013, S. ZorzalAlmeida \& D.C. Bicudo (phytoplankton: SP469281, SP469282, SP469283, SP469284); Mairiporã, Paiva Castro, 19-VII-2013, S. Zorzal-Almeida \& D.C. Bicudo (phytoplankton: SP469303, SP469304); Nazaré Paulista, Atibainha, 24-I-2013, S. ZorzalAlmeida \& D.C. Bicudo (phytoplankton: SP469275); Nazaré Paulista, Atibainha, 25-VII-2013, S. ZorzalAlmeida \& D.C. Bicudo (phytoplankton: SP469297); Piracaia, Cachoeira, 21-II-2013, S. Zorzal-Almeida \& D.C. Bicudo (phytoplankton: SP469270, SP469271, SP469272, SP469273, SP469274); Piracaia, Cachoeira, 26-VII-2013, S. Zorzal-Almeida \& D.C. Bicudo (phytoplankton: SP469292, SP469293, SP469294).

\section{Achnanthidium sp.}

Figures 80-87

Valves linear-lanceolate with rounded to subrostrate ends; raphe valves with narrow and linear axial area. L: 11.5-18.0 $\mu \mathrm{m}$; W: 2.4-2.8 $\mu \mathrm{m}$; inconspicuous striae.

The principal feature to distinguish this species from A. minutissimum is its narrower width measures (Hlúbiková et al. 2011; W: 2.5-3.1 $\mu \mathrm{m}$ ). Further studies, such as SEM are necessary to a detailed analysis and to investigate whether Achnanthidium sp. 1 represents a new species. In the present study, this species was recorded in surface sediment of four reservoirs ranging from oligo- to eutrophic conditions (Santa Helena, Itupararanga, Ipaneminha and Hedberg, maximum abundance: $6.3 \%$ ), and in the phytoplankton of the mesotrophic reservoir Ipaneminha (maximum abundance: $0.8 \%$ ), presenting higher frequency in mesotrophic waters (17.1\%).

Examined material: BRASIL. São PAUlo: Iperó, Hedberg, 26-VIII-2014, E. Bartozek \& D.C. Bicudo (surface sediment: SP469240, SP469241); Sorocaba, Ipaneminha, 21-VIII-2014, E. Bartozek \& D.C.
Bicudo (phytoplankton: SP469506, SP469507; surface sediment: SP469237); Sorocaba, Itupararanga, 25-VIII-2014, E. Bartozek \& D.C. Bicudo (surface sediment: SP469236); Sorocaba, Itupararanga, 29-VIII-2014, E. Bartozek \& D.C. Bicudo (surface sediment: SP469234); Votorantim, Santa Helena, 21-VIII-2014, E. Bartozek \& D.C. Bicudo (surface sediment: SP469229, SP469230).

\section{Brachysiraceae Mann}

\section{Brachysira Kützing}

\section{Brashysira sp.}

Figures 88-91

Valves rhombic-lanceolate with ends strongly capitate; axial area is narrow and linear, central area is small and rhombic; raphe is filiform; striae are slightly radiated in middle portion becoming parallel toward the ends. L: 13.5-27.0 $\mu \mathrm{m}$; W: 3.2-5.0 $\mu \mathrm{m}$; S: 32-35 in $10 \mu \mathrm{m}$.

Brachysira sp. is similar to Brachysira microcephala (Grunow) Compère "morphotype 2" presented by Siver et al. (2005) due to the distinctly capitate ends. However, Brachysira sp. differs due to its shorter width and valves becoming narrower toward the ends. Besides, Brachysira sp. maintains the valve shape (capitate) in smaller specimens. In the present study, this taxon was recorded in surface sediment of three reservoirs (Atibainha, Paiva Castro and Tatu, maximum abundance: 5.3\%), however, it exhibited higher frequency in oligotrophic samples (16.0\%).

Examined material: BRASIL. SÃo PAUlo: Limeira, Tatu, 18-VII-2013, S. Zorzal-Almeida \& D.C. Bicudo (surface sediment: SP469268, SP469269); Mairiporã, Paiva Castro, 19-VII-2013, S. Zorzal-Almeida \& D.C. Bicudo (surface sediment: SP469260, SP469261, SP469262); Nazaré Paulista, Atibainha, 25-VII-2013, S. Zorzal-Almeida \& D.C. Bicudo (surface sediment: SP469254, SP469255, SP469256, SP469257, SP469258).

Sellaphoraceae Mereschkowsky

Sellaphora Mereschkowsky

*Sellaphora rostrata (Hustedt) Johansen, Archiv für Hydrobiologie Supplement 150 (Algological Studies 111), p. 17-44, 2004.

Figures 92-93 
L: $17.5-29.0 \mu \mathrm{m} ; \mathrm{W}: 6.3-8.0 \mu \mathrm{m}$; $\mathrm{S}: 19-25$ in $10 \mu \mathrm{m}$.

This taxon belongs to the Sellaphora pupula complex. Nevertheless, S. rostrata can be distinguished from $S$. pupula because the first species presents distinct capitate ends (Johansen et al. 2004). It can be found in circumneutral to alkaline and eutrophic environments (Moro \& Fürstenberger 1997). In the present study, it was found in the surface sediment of two oligoand mesotrophic reservoirs (Paiva Castro and Tatu, maximum abundance: 5.4\%), and in the phytoplankton of four reservoirs ranging from oligo- to eutrophic conditions (Cachoeira, Paiva Castro, Tatu and Salto Grande, maximum abundance: $2.6 \%$ ), showing higher frequency in mesotrophic samples $(17.1 \%)$.

Examined material: BRASIL. São PAULO: Americana, Salto grande, 20-IX-2013, S. Zorzal-Almeida \& D.C. Bicudo (phytoplankton: SP469308); Limeira, Tatu, 18-IV-2013, S. Zorzal-Almeida \& D.C. Bicudo (phytoplankton: SP469290); Limeira, Tatu, 18-VII2013, S. Zorzal-Almeida \& D.C. Bicudo (phytoplankton: SP469311, SP469312, SP469313; surface sediment: SP469267, SP469268, SP469269); Mairiporã, Paiva Castro, 18-I-2013, S. Zorzal-Almeida \& D.C. Bicudo (phytoplankton: SP469283); Mairiporã, Paiva Castro, 19-VII-2013, S. Zorzal-Almeida \& D.C. Bicudo (phytoplankton: SP469303; surface sediment: SP469261, SP469262); Piracaia, Cachoeira, 21-II-2013, S. ZorzalAlmeida \& D.C. Bicudo (phytoplankton: SP469270); Piracaia, Cachoeira, 26-VII-2013, S. Zorzal-Almeida \& D.C. Bicudo (phytoplankton: SP469292).

\section{Bacillariaceae Ehrenberg}

\section{Nitzschia Hassal}

**Nitzschia perminuta (Grunow) Peragallo, Le Catalogue Général des Diatomées [issued in fascicles at various dates] vol. 2, p. 672, 1903. Figures 94-95

L: $17.0-26.0 \mu \mathrm{m} ; \mathrm{W}: 2.1-4.0 \mu \mathrm{m} ; \mathrm{S}: 25-26$ in $10 \mu \mathrm{m}$; F: $10-17$ in $10 \mu \mathrm{m}$.

$N$. perminuta can be distinguished from Nitzchia amphibia Grunow because the second species presents evident central nodule, which is lacking in $N$. perminuta, and prominent and distinctly punctate striae (Kociolek 2011a). N. perminuta has been found in alkaline and eutrophic waters (Van Dam et al. 1994) and in the epiphyton of a mesotrophic subtropical reservoir (Marra et al. 2016). However,
$N$. cf. perminuta was found in slightly acidic to circumneutral ponds with low nutrients concentration (Siver et al. 2005). In the present study, it occurred in the surface sediment of two oligotrophic reservoirs (Atibainha and Paiva Castro, maximum abundance: $2.0 \%$, with a frequency of $8.0 \%$ in the samples.

Examined material: BRASIL. São PaUlo: Mairiporã, Paiva Castro, 19-VII-2013, S. Zorzal-Almeida \& D.C. Bicudo (surface sediment: SP469260, SP469261); Nazaré Paulista, Atibainha, 25-VII-2013, S. Zorzal-Almeida \& D.C. Bicudo (surface sediment: SP469253, SP469254).

*Nitzschia semirobusta Lange-Bertalot, Bibliotheca Diatomologica 27, pl. 120, figs. 3-21, pl. 122, fig. 7, pl. 123, figs. 1-7, 1993.

Figures 96-101

L: $8.0-29.0 \mu \mathrm{m} ; \mathrm{W}: 3.5-5.0 \mu \mathrm{m}$; $\mathrm{S}: 16-18$ in 10 $\mu \mathrm{m}$; F: $7-8$ in $10 \mu \mathrm{m}$.

This species can be confused with Nitzschia amphibia Grunow due to the same valve shape. However, $N$. semirobusta can be distinguished by its more extended fibulae (Lange-Bertalot 1993). The taxon also resembles Denticula kuetzingii Grunow, nevertheless, the fibulae of the second species is extended from margin to margin with a similar thickness, while the fibulae of $N$. semirobusta decrease in thickness as they extend across the valve from one margin to another (Underwood 2017). In the present study, it was observed in surface sediment of five reservoirs ranging from oligo- to eutrophic conditions (Atibainha, Cachoeira, Paiva Castro, Tatu and Salto Grande, maximum abundance: $4.3 \%$ ), and in phytoplankton of four reservoirs ranging from oligoto mesotrophic conditions (Atibainha, Cachoeira, Paiva Castro and Tatu, maximum abundance: 5.0\%). Higher frequency occurred in oligotrophic conditions (74.0\%).

Examined material: BRASIL. São PAUlo: Limeira, Tatu, 18-IV-2013, S. Zorzal-Almeida \& D.C. Bicudo (phytoplankton: SP469289, SP469290, SP469291); Limeira, Tatu, 18-VII-2013, S. Zorzal-Almeida \& D.C. Bicudo (phytoplankton: SP469311, SP469312; surface sediment: SP469269); Mairiporã, Paiva Castro, 18-I-2013, S. Zorzal-Almeida \& D.C. Bicudo (phytoplankton: SP469282, SP469283); Mairiporã, Paiva Castro, 19-VII-2013, S. Zorzal-Almeida \& D.C. Bicudo (phytoplankton: SP469303, SP469304, SP469305; surface sediment: SP469259, SP469260, SP469261, SP469262); Nazaré Paulista, Atibainha, 24-I-2013, S. Zorzal-Almeida \& D.C. Bicudo 
(phytoplankton: SP469275, SP469276, SP469277, SP469278, SP469279, SP469280); Nazaré Paulista, Atibainha, 25-VII-2013, S. Zorzal-Almeida \& D.C. Bicudo (phytoplankton: SP469297, SP469298, SP469300, SP469302; surface sediment: SP469253, SP469254, SP469255, SP469257, SP469258); Piracaia, Cachoeira, 21-II-2013, S. Zorzal-Almeida \& D.C. Bicudo (phytoplankton: SP469270, SP469271, SP469272, SP469273, SP469274); Piracaia, Cachoeira, 26-VII-2013, S. Zorzal-Almeida \& D.C. Bicudo (phytoplankton: SP469292, SP469294, SP469295, SP469296; surface sediment: SP469248, SP469249, SP469250, SP469251, SP469252).

\section{Nitzschia sp. 1}

Figures 102-109

Valves lanceolate; apices acutely rounded; striae parallel; fibulae equally spaced; central nodule absent. L: $16.5-32.2 \mu \mathrm{m}$; W: 3.8-4.5 $\mu \mathrm{m}$; S: 17-19 in $10 \mu \mathrm{m}$; F: $8-9$ in $10 \mu \mathrm{m}$.

Nitzschia sp. 1 is similar to Nitzschia amphibia Grunow and $N$. fonticola (Grunow) Grunow in relation to the valve shape and conspicuous striae (Kociolek 2011a, 2011b). However, Nitzschia sp. 1 doesn't present central nodule, which is evident in the two other species (Trobajo et al. 2006). Furthermore, $N$. fonticola exhibit higher striae density (S: 28-30 in $10 \mu \mathrm{m}$ ) than Nitzschia sp. 1 (Trobajo et al. 2006). In this study it was found in surface sediment of three reservoirs ranging from meso- to eutrophic conditions (Itupararanga, Ipaneminha and Hedberg, maximum abundance: $7.0 \%$ ), and in phytoplankton of the same reservoirs (maximum abundance: 1.2\%). Higher frequency occurred in mesotrophic samples $(25.7 \%)$.

Examined material: BRASIL. São PAulo: Iperó, Hedberg, 12-III-2014, E. Bartozek \& D.C. Bicudo (phytoplankton: SP469508, SP469509, SP469510); Iperó, Hedberg, 26-VIII-2014, E. Bartozek \& D.C. Bicudo (phytoplankton: SP469511, SP469512; surface sediment: SP469240, SP469241, SP469242); Sorocaba, Ipaneminha, 25-II-2014, E. Bartozek \& D.C. Bicudo (phytoplankton: SP469502); Sorocaba, Ipaneminha, 21VIII-2014, E. Bartozek \& D.C. Bicudo (phytoplankton: SP469505; surface sediment: SP469237, SP469239); Sorocaba, Itupararanga, 21-II-2014, E. Bartozek \& D.C. Bicudo (phytoplankton: SP469495); Sorocaba, Itupararanga, 25-VIII-2014, E. Bartozek \& D.C. Bicudo (surface sediment: SP469236).

Nitzschia sp. 2

Figures 110-112
Valves linear-lanceolate; ends expanded, subcapitate; striae parallel becoming slightly curved toward the ends, almost inconspicuous; fibulae equally spaced. L: 19.0-20.5 $\mu \mathrm{m}$; W: 3.5-4.5 $\mu \mathrm{m}$; S: 23-26 in $10 \mu \mathrm{m}$; F: $10-13$ in $10 \mu \mathrm{m}$.

Nitzschia sp. 2 resembles $N$. bryophila (Hustedt) Hustedt because of their similar valve shape. Nevertheless, $N$. bryophila is wider (W: $5.0 \mu \mathrm{m}$ ), presents higher striae density (S: 31-33 in $10 \mu \mathrm{m}$ ) and more prominent ends (Simonsen 1987). In the present study, it was found in surface sediment of mesotrophic reservoir Tatu (maximum abundance: $20.2 \%$ ), and in the phytoplankton of the same reservoir (maximum abundance: $3.3 \%$ ), with a frequency of $11.4 \%$ in the mesotrophic samples.

Examined material: BRASIL. São PAULO: Limeira, Tatu, 18-VII-2013, S. Zorzal-Almeida \& D.C. Bicudo (phytoplankton: SP469311, SP469312, SP469313; surface sediment: SP469267, SP469268, SP469269).

\section{Nitzschia sp. 3}

Figures 113-127

Valves linear to linear-lanceolate, frustules rectangular in girdle view; ends varying from rounded to slightly subrostrate; fibulae irregularly spaced. L: 38.3-62.7; W: 4.0-4.7; inconspicuous striae; F: 11-14 in $10 \mu \mathrm{m}$.

This species is similar to Nitzschia linearis (Agardh) Smith due to its linear-lanceolate valves (Kociolek 2011c), however, Nitzschia sp. 3 presents more attenuated ends. Nitzschia sp. 3 is also similar to $N$. gracilis Hantzsch. Nevertheless, $N$. gracilis exhibits larger valve variation, with individuals ranging from around $30.0 \mu \mathrm{m}$ to more than $100.0 \mu \mathrm{m}$ (Lange-Bertalot 1976). In this study, Nitzschia sp. 3 occurred in phytoplankton of hypereutrophic reservoir Barra Bonita (maximum abundance: 12.0\%), with a frequency s of $18.4 \%$ (eu- and hypereutrophic samples).

Examined material: BRASIL. São PAulo: Barra Bonita, Barra Bonita, 20-III-2014, E. Bartozek \& D.C. Bicudo (phytoplankton: SP469514, SP469515, SP469516, SP469517); Barra Bonita, Barra Bonita, 29-VII-2014, E. Bartozek \& D.C. Bicudo (phytoplankton: SP469519, SP469520, SP469521).

Summarizing, from a total of 78 taxa recorded in surface sediments and phytoplankton samples, our study added six new diatom species to the Brazilian flora and beyond those, six for São Paulo State. 
Furthermore, we registered other ten possible new taxa for science. Eight taxa occurred exclusively in the surface sediment (Achnanthidium lineare, Brachysira sp., Fragilaria parva, Gomphonema parvulum, Hantzschia amphioxys, Navicula symmetrica, Nitzschia perminuta and Sellaphora nigri (see table 4). Other eighteen taxa only occurred in the phytoplankton community (Achnanthidium exiguum, A. cf. macrocephalum, Achnanthidium tropicocatenatum, Aulacoseira veraluciae, Brachysira brebissonii, Discostella pseudostelligera, Encyonopsis thienemannii, Eunotia desmogonioides, E. meridiana, Fragilaria crotonensis, F. spectra, Geissleria punctifera, Gomphonema hawaiense, Nitzschia amphibia, N. gracilis, N. palea var. debilis, Nitzschia sp. 3 and Ulnaria acus). Three species were exclusively recorded in oligotrophic conditions (Navicula symmetrica, Nitzschia perminuta and Sellaphora nigri), Eunotia desmogonioides was only found in mesotrophic conditions and four species occurred only in eutrophic waters (Aulacoseira veraluciae, Brachysira brebissonii, Nitzschia sp. 3 and Placoneis exigua).

Regarding the twelve species registered as new records for Brazil and São Paulo State, five of them presented wide distribution in relation to the trophic conditions, occurring from oligo- to eutrophic conditions (Achnanthidium jackii, A. cf. macrocephalum, Cymbella affinis var. neoprocera, Fragilaria grunowii and Sellaphora rostrata). Other four taxa occurred in oligo-mesotrophic conditions (Encyonopsis thienemannii, Gomphonema naviculoides, Nitzschia perminuta and N. semirobusta), while Achnanthidium lineare occurred in mesoeutrophic and Placoneis exigua was only recorded in eutrophic conditions. Two of the new records were exclusively found in the surface sediment (Achnanthidium lineare and Nitzschia perminuta) and other two only in the phytoplankton (Achnanthidium cf. macrocephalum and Encyonopsis thienenmannii). Finally, this study increased the information on the ecology and distribution of these species, particularly in tropical reservoirs, and highlights the need of floristic surveys to improve the knowledge of biodiversity of freshwater tropical diatoms.

\section{Acknowledgements}

This study was carried out within the framework of the AcquaSed project supported by funds from
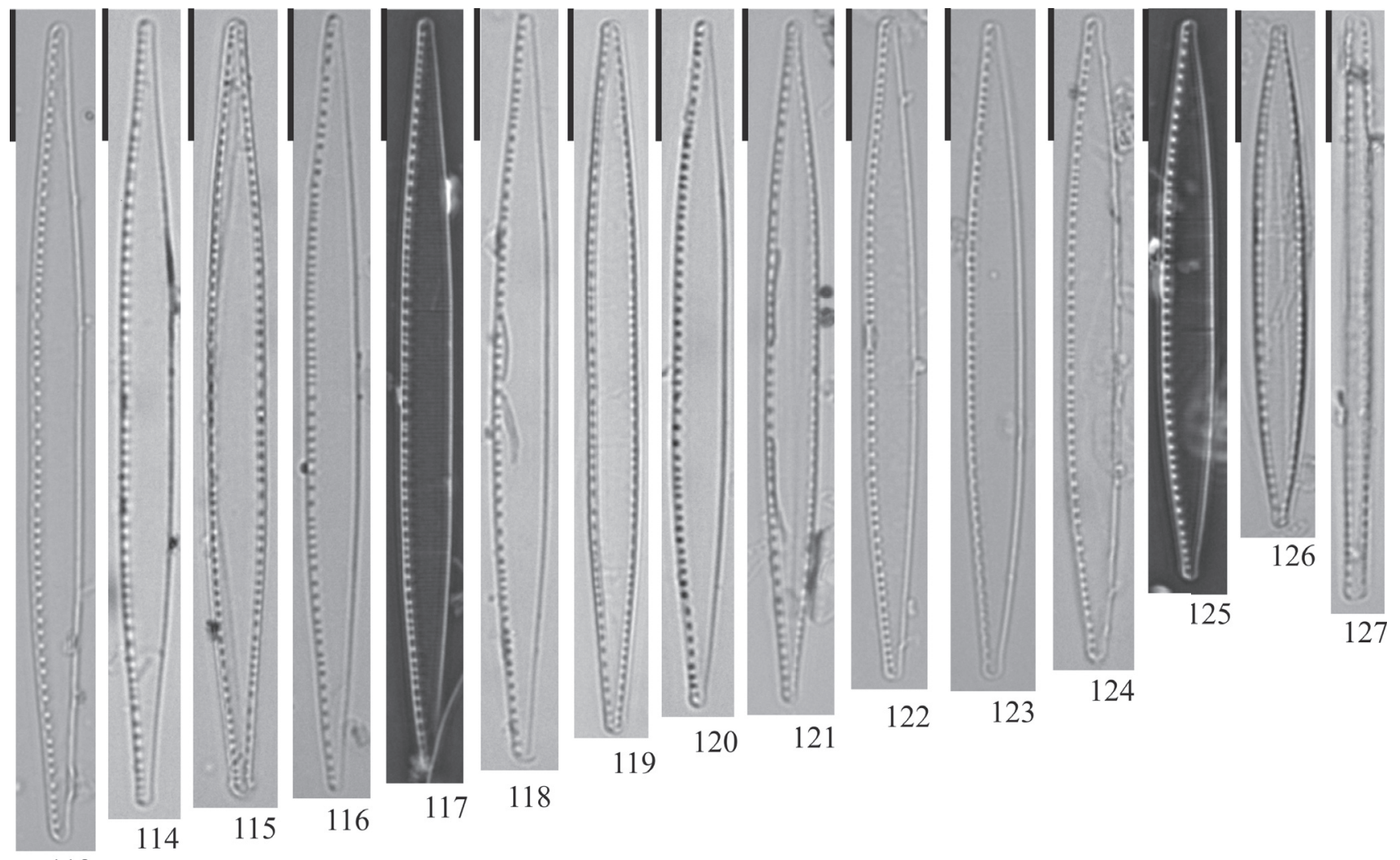

113

Figures 113-127. Surface sediment and planktonic diatoms. 113-127. Nitzschia sp. 3 (Fig. 127. Lateral view). Scale bar $=10 \mu \mathrm{m}$. 
FAPESP (Fundação de Amparo à Pesquisa do Estado de São Paulo, AcquaSed Project, nº 2009/53898-9). ECRB and SZA thanks their doctoral fellowship (FAPESP process 2013/14337-7 and 2013/23703-7, respectively). Funding was also provided by $\mathrm{CNPq}$, Conselho Nacional de Desenvolvimento Científico e Tecnológico (Grant 310404/2016-9 to DCB). We appreciate the valuable assistance of personnel from the agency in charge of the public water supply in São Paulo - SABESP (Companhia de Saneamento do Estado de São Paulo), Barco Escola da Natureza Association, Companhia Energética Salto do Lobo, Floresta Nacional do Ipanema and Votorantin Energia for their valuable logistical support during the fieldwork. We deeply thank all the students from the Department of Ecology involved in field and laboratory work and those who collaborated in the construction of the AcquaSed database. We are also grateful to Prof. William de Queiróz (Universidade de Guarulhos, Laboratório de Geoprocessamento) for the study area map.

\section{Literature cited}

Almeida, P.D. \& Bicudo, D.C. 2014. Diatomáceas planctônicas e de sedimentos superficiais em represas de abastecimento da Região Metropolitana de São Paulo, Sudeste do Brasil. Hoehnea 41: 187-207.

Almeida, P.D., Wetzel, C.E., Morales, E.A., Ector, L. \& Bicudo D.C. 2015. Staurosirella acidophila sp. nov., a new araphid diatom (Bacillariophyta) from southeastern Brazil: ultrastructure, distribution and autecology. Cryptogamie, Algologie 36: 255-270.

Almeida, P.D., Morales, E.A., Wetzel, C.E., Ector, L. \& Bicudo D.C. 2016. Two new diatoms in the genus Fragilaria Lyngbye (Fragilariophyceae) from tropical reservoirs in Brazil and comparison with type material of F. tenera. Phytotaxa 246: 163-183.

APHA - American Public Health Association. 2005. Standard Methods for the Examination of Water and Wastewater, $21^{\text {st }}$ Edition, American Public Health Association, Washington, DC.

Bartozek, E.C.R., Bueno, N.C., Ludwig, T.A.V., Tremarin, P.I., Nardelli, M.S. \& Rocha, A.C.R. 2013. Diatoms (Bacillariophyceae) of Iguaçu National Park, Foz do Iguaçu, Brazil. Acta Botanica Brasilica 27: 108-123.

Battarbee, R.W., Jones, V.J., Flower, R.J., Cameron, N.G., Bennion, H., Carvalho, L. \& Juggins, S. 2001. Diatoms. In: J.P. Smol, H.J.B. Birks \& W.M. Last (eds.). Tracking environmental change using lake sediments, v. 3: terrestrial, algal, and siliceous indicators. Kluwer, Dordrecht, pp. 155-202.
Bennion, H., Kelly, M.G., Juggins, S., Yallop, M.L., Burgess, A. Jamieson, J. \& Krokowski, J. 2014. Assessment of ecological status in UK lakes using benthic diatoms. Freshwater Science 33: 639-654.

Bere, T. \& Tundisi, J.G. 2010. Epipsammic diatoms in streams influenced by urban pollution, São Carlos, SP, Brazil. Brazilian Journal of Biology 70: 921-930.

Bertolli, L.M., Tremarin, P.I. \& Ludwig, T.A.V. 2010. Diatomáceas perifíticas em Polygonum hydropiperoides Michaux, reservatório do Passaúna, Região Metropolitana de Curitiba, Paraná, Brasil. Acta Botanica Brasilica 24: 1065-1081.

Besse-Lototskaya, A., Verdonschot, P.F.M., Coste, M. \& Van de Vijver, B. 2011. Evaluation of European diatom trophic índices. Ecological Indicators 11: 456-467.

Bicudo, D.C., Tremarin, P.I., Almeida, P.D., ZorzalAlmeida, S., Wengrat, S., Faustino, S.B., Costa, L.F., Bartozek, E.C.R., Rocha, A.C.R., Bicudo, C.E.M. \& Morales, E.A. 2016. Ecology and distribution of Aulacoseira species (Bacillariophyta) in tropical reservoirs from Brazil. Diatom Research 31: 199-215.

Blanco, S., Cejudo-Figueiras, C., Alvarez-Blanco, I., Van Donk, E., Gross, E.M., Hansson, L., Irvine, K., Jeppesen, E., Kairesalo, T., Moss, B., Nôges, T. \& Bécares, E. 2014. Epiphytic diatoms along environmental gradients in western European shallow lakes. Clean - Soil, Air, Water 42: 229-235.

California Academy of Sciences. 2012. Diatom Collection. California. Available in http://research.calacademy.org/ $\mathrm{izg} /$ research/diatom (access in 01-II-2017).

Carneiro, L.A. \& Bicudo, D.C. 2007. O gênero Lemnicola (Bacillariophyceae) no Estado de São Paulo, Brasil. Hoehnea 34: 253-259.

Ceballos, G., Ehrlich, P.R., Barnosky, A.D., García, A., Pringle, R.M. \& Palmer, T.M. 2015. Accelerated modern human-induced species losses: entering in the sixth mass extinction. Science Advances 1: e1400253.

Dudgeon, D., Arthington, A.H., Gessner, M.O., Kawabata, Z., Knowler, D.J., Lévêque, C., Naiman, R.J., Pieur-Richard, A., Soto, D., Stiassny, M.L.J. \& Sullivan, C.A. 2006. Freshwater biodiversity: importance, threats, status and conservation challenges. Biology Reviews 81: 163-182.

Faustino, S.B., Fontana, L., Bartozek, E.C.R., Bicudo, C.E.M. \& Bicudo, D.C. 2016. Composition and distribution of diatom assemblages from core and surface sediments of a water supply reservoir in Southeastern Brazil. Biota Neotropica 16: 1-23.

Ferreira, K.S.M. \& Bicudo, C.E.M. 2017. Criptógamos do Parque Estadual das Fontes do Ipiranga, São Paulo, SP. Algae, 42: Bacillariophyceae (Surirellales). Hoehnea 44: $10-28$.

Fontana, L. \& Bicudo, D.C. 2009. Diatomáceas (Bacillariophyceae) de sedimentos superficiais dos reservatórios em cascata do Rio Paranapanema (SP/ PR, Brasil): Coscinodiscophyceae e Fragilariophyceae. Hoehnea 36: 375-386. 
Fontana, L. \& Bicudo, D.C. 2012. Biodiversidade e distribuição das diatomáceas (Bacillariophyceae) de sedimentos superficiais nos reservatórios em cascata do Rio Paranapanema (SP/PR, Brasil). Hoehnea 39: 587-612.

Garcia, M. 2010. A new diatom genus: Rimoneis M.Garcia (Fragilariaceae, Bacillariophyceae): a new hyaline araphid genus based on observations of frustules from Southern Brazil. Diatom 26: 1-9.

Hamsher, S.E., Graeff, C.L., Stepanek, J.G. \& Kociolek, P. 2014. Frustular morphology and polyphyly in freshwater Denticula (Bacillariophyceae) species, and the description of Tetralunata gen. nov. (Epithemiaceae, Rhopalodiales). Plant Ecology and Evolution 147: 346-365.

Hlúbiková, D., Ector, L. \& Hoffmann, L. 2011. Examination of the type material of some diatom species related to Achnanthidium minutissimum (Kütz.) Czarn. (Bacillariophyceae) Algological Studies 136/137: 19-43.

Hustedt, F. 1937. Systematische und ökologische Untersuchungen über die Diatomeen-Flora von Java, Bali und Sumatra nach dem Material der Deutschen Limnologischen Sunda-Expedition. Archiv für Hydrobiologie (Supplement) 15: 178-295.

Johansen, J.R., Lowe, R., Gomez, S.R., Kociolek J.P. \& Marakosky, S.A. 2004. New algal species records for the Great Smoky Mountains National Park, U.S.A., with an annotated checklist of all reported algal species for the park. Algological Studies 111: 17-44.

Julius, M.L. \& Theriot, E.C. 2010. The diatoms: a primer. In: J.P. Smol \& E.F. Stoermer (eds.). The diatoms: applications for the environmental and earth sciences. 2 ed. Cambridge University Press: Cambridge.

Kociolek, P. 2011a. Nitzschia amphibia. In: Diatoms of the United States. Available in https://westerndiatoms. colorado.edu/taxa/species/nitzschia_amphibia (access in 24-VII-2017).

Kociolek, P. 2011b. Nitzschia fonticola. In Diatoms of the United States. Available in http://westerndiatoms. colorado.edu/taxa/species/nitzschia_fonticola (accessed in 18-XII-2016).

Kociolek, P. 2011c. Nitzschia linearis. In: Diatoms of the United States. Available in https://westerndiatoms. colorado.edu/taxa/species/nitzschia_linearis (access in 06-IV-2017).

Krammer, K. 1997. Die cymbelloiden Diatomeen, 1: Allgemeines und Encyonema part. Bibliotheca Diatomologica, v. 36.

Krammer, K. 2002. Diatoms of the European inland waters and comparable habitats. Diatoms of Europe, v. 3. Koeltz Scientific Books, Königstein.

Lamparelli, M.C. 2004. Graus de trofia em corpos d'água do Estado de São Paulo: Avaliação dos métodos de monitoramento. Tese de Doutorado, Universidade de São Paulo, São Paulo.
Lange-Bertalot, H. 1976. Eine revision zur taxonomie der Nitzschiae lanceolatae Grunow. Nova Hedwigia 28: 253-307.

Lange-Bertalot, H. 1993. 85 Neue Taxa und über 100 weitere neu definierte Taxa ergänzend zur Süßwasserflora von Mitteleuropa. Bibliotheca Diatomologica, v. 27, pp.1-454.

Lange-Bertalot, H. 2000. Diatoms of the Andes: from Venezuela to Patagonia/Tierra del Fuego and two new additional contributions. In: H. Lange-Bertalot (ed.). Iconographia Diatomologica, v. 9, pp. 1-673.

Lange-Bertalot, H., Bak, M. \& Witkowski, A. 2011. Diatoms of the European Inland Water and Comparable Habitats. Eunotia and some related genera. In: H. LangeBertalot (ed.). Diatoms of Europe, v. 6, pp. 1-747.

Lange-Bertalot, H. \& Ulrich, S. 2014. Contributions to the taxonomy of needle-shaped Fragilaria and Ulnaria species. Lauterbornia 78: 1-73.

Ludwig, T.A.V., Tremarin, P.I., Neiva, T.F., Coquemala, V. \& Piccinini, C. 2005. Diatomáceas (Ochrophyta) dos lagos do Jardim Botânico, Curitiba, Paraná. In: X Reunião Brasileira de Ficologia. Formação de ficólogos: um compromisso com a sustentabilidade dos recursos aquáticos, Sociedade Brasileira de Ficologia, pp. 301-323.

Mann, D.G. \& Vanormelingen, P. 2013. An inordinate fondness? The number, distributions, and origins of diatom species. The Journal of Eukaryotic Microbiology 60: 414-420.

Marra, R.C., Tremarin, P.I., Algarte, V.M. \& Ludwig, T.V. 2016. Epiphytic diatoms (Diatomeae) from Piraquara II urban reservoir, Paraná State. Biota Neotropica 16: e20160200.

Marquardt, G.C. \& Bicudo, C.E.M. 2014. Criptógamos do Parque Estadual das Fontes do Ipiranga, São Paulo, SP. Algas 36: Bacillariophyceae (Cymbellales). Hoehnea 41: 209-246.

Marquardt, G.C., Rocha, A.C.R., Wetzel, C.E., Ector, L. \& Bicudo, C.E.M. 2016. Encyonema aquasedis sp. nov. and Kurtkrammeria salesopolensis sp. nov.: two new freshwater diatom species (Cymbellales, Bacillariophyceae) from an oligotrophic reservoir in southeastern Brazil. Phytotaxa 247: 62-74.

Medlin, L.K. \& Kaczamarska, I. 2004. Evolution of the diatoms V. Morphological and cytological support for the major clades and taxonomic revision. Phycologia 43: $245-270$.

Metzeltin, D. \& Lange-Bertalot, H. 1998. Tropical Diatoms of South America I. About 700 predominantly rarely know or new taxa representative of the neotropical flora. In: H. Lange-Bertalot, H. (ed.). Iconographia Diatomologica, v. 5, pp. 1-695.

Morales, E.A. 2001. Morphological studies in selected fragilarioid diatoms (Bacillariophyceae) from Connecticut waters (U.S.A.). Proceedings of the Academy of Natural Sciences pf Philadelphia 151: 105-120. 
Morales, E.A., Guerrero, J.M., Wetzel, C.E., Sala, S. \& Ector, L. 2013. Unraveling the identity of Fragilaria pinnata Ehrenberg and Staurosira pinnata Ehrenberg: research in progress on a convoluted story. Cryptogamie, Algologie 34: 89-102.

Moro, R.S. \& Fürstenberger, C.B. 1993. Diatomáceas (Bacillariophyceae) da Lagoa Dourada (Parque Estadual de Vila Velha), Paraná, Brasil. Acta Biológica Paranaense 22: 15-30.

Nardelli, M.S., Bueno, N.C., Ludwig, T.A.V., Tremarin, P.I. \& Bartozek, E.C.R. 2014. Coscinodiscophyceae and Fragilariophyceae (Diatomeae) in the Iguaçu River, Paraná, Brazil. Acta Botanica Brasilica 28: 127-140.

Passy, S.I. 2007. Diatom ecological guilds display distinct and predictable behavior along nutrient and disturbance gradients in running waters. Aquatic Botany 86: 171-178.

Potapova, M. \& Hamilton, P. 2007. Morphological and ecological variation within the Achnanthidium minutissimum (Bacillariophyceae) species complex. Journal of Phycology 43: 561-575.

Reichardt, E. 2015. Gomphonema gracile Ehrenberg sensu stricto et sensu auct. (Bacillariophyceae): A taxonomic revision. Nova Hedwigia 101: 367-393.

Ribeiro, F.C.P., Senna, C.S.F. \& Torgan, L.C. 2008. Diatomáceas em sedimentos superficiais na planície de maré da Praia de Itupanema, Estado do Pará, Amazônia. Rodriguésia 59: 309-324.

Round, F.E., Crawford, R.M. \& Mann, D.G. 1990. The diatoms. Biology and morphology of the genera. Cambridge University Press, New York.

Sartory, D.P. \& Grobbelaar, J.U. 1984. Extraction of chlorophyll a from freshwater phytoplankton for spectrophotometric analysis. Hydrobiologia 114: 177-187.

Silva, P.D.A. 2017. Taxonomia e ecologia de diatomáceas arrafídeas (Fragilariales, Bacillariophyta) no estado de São Paulo, Brasil. Tese de Doutorado, Instituto de Botânica, São Paulo.

Silva, A.M., Ludwig, T.A.V., Tremarin, P.I. \& Vercellino, I.S. 2010. Diatomáceas perifíticas em um sistema eutrófico brasileiro (Reservatório do Iraí, estado do Paraná). Acta Botanica Brasilica 24: 997-1016.

Silva, W.J., Jahn, R., Ludwig, T.A.V., Hinz, F. \& Menezes, M. 2015. Typification and taxonomic status re-evaluation of 15 taxon names within the species complex Cymbella affinis/tumidula/turgidula (Cymbellaceae, Bacillariophyta). PhytoKeys 53: 1-25.

Simonsen, R. 1987. Atlas and Catalogue of the Diatom types of Friedrich Hustedt 2: 476-477. Berlin, J. Cramer.
Siver, P.A., Hamilton, P.B., Stachura-Suchoples, K. \& Kociolek, K.P. 2005. Diatoms of North America: the freshwater flora of Cape Cod, Massachusetts, U.S.A. Iconographia Diatomologica 14: 1-463.

Souza, M.G.M. \& Senna, P.A.C. 2009. Diatomáceas epilíticas da subordem Sellaphorineae do rio Monjolinho, São Carlos, SP, Brasil. Acta Botanica Brasilica 23: 618-629.

Sovereign, H.E. 1958. The diatoms of crater lake, Oregon. Transactions of the American Microscopical Society 77: 96-134.

Strayer, D.L. \& Dudgeon, D. 2010. Freshwater biodiversity conservation: recent progress and future challenges. Journal of the North American Benthological Society 29: 344-358.

Taylor, J.C., Harding, W.R. \& Archibald, G.M. 2007. An Illustrated Guide to Some Common Diatom Species from South Africa. WRC Report No. TT 282/07. Water Research Commission, Pretoria, South Africa.

Trobajo, R., Mann, D.G., Chepurnov, V.A., Clavero, A. \& Cox, E. 2006. Taxonomy, life cycle, and auxosporulation of Nitzschia fonticola (Bacillariophyta). Journal of Phycology 42: 1353-1372.

Tuji, A. \& Williams, D.M. 2008. Typification and type examination of Synedra familiaris Kiitz. and related taxa. Diatom 24: 25-29.

Underwood, D. 2013. Nitzschia semirobusta. In Diatoms of the United States. Available in https://westerndiatoms. colorado.edu/taxa/species/nitzschia_semirobusta (access in 29-XI-2017).

Van Dam, H., Mertens, A. \& Sinkeldam, J. 1994. A coded checklist and ecological indicator values of freshwater diatoms from the Netherlands. Netherlands Journal of Aquatic Ecology 28: 117-133.

Van de Vijver B., Ector L., Beltrami, M.E., Haan M., Falasco E., Hlúbiková D., Jarlman A., Kelly M., Novais M.H. \& Wojtal, A.Z. 2011. A critical analysis of the type material of Achnanthidium lineare W. Sm. (Bacillariophyceae). Algological Studies 136/137: 167-191.

Wetzel, C.E. \& Ector, L. 2015. Taxonomy and ecology of Fragilaria microvaucheriae sp. nov. and comparison with the type materials of $F$. uliginosa and $F$. vaucheriae. Cryptogamie, Algologie 36: 271-289.

Wojtal, A., Ector, L., Van de Vijver, B., Morales, E.A., Blanco, S., Piatek J. \& Smieja A. 2011. The Achnanthidium minutissimum complex (Bacillariophyceae) in southern Poland. Algological Studies 137/137: 211-238. 